\title{
The homotopy category of flat modules, and Grothendieck duality
}

\author{
Amnon Neeman ${ }^{\star}$ \\ Centre for Mathematics and its Applications, Mathematical Sciences Institute, \\ John Dedman Building, The Australian National University, Canberra, ACT 0200, \\ Australia (e-mail: Amnon. Neeman@anu .edu.au)
}

Oblatum 19-VII-2007 \& 5-III-2008

Published online: 20 August 2008 - (C) Springer-Verlag 2008

Dedicated with gratitude to my PhD thesis adviser, David Mumford, on his 70th birthday

Abstract. Let $R$ be a ring. We prove that the homotopy category $\mathbf{K}(R$-Proj $)$ is always $\aleph_{1}$-compactly generated, and, depending on the ring $R$, it may or may not be compactly generated. We use this to give a description of $\mathbf{K}(R$-Proj) as a quotient of $\mathbf{K}(R$-Flat $)$. The remarkable fact is that this new description of $\mathbf{K}(R$-Proj) generalizes to non-affine schemes; this will appear in Murfet's thesis.

\section{Contents}

0 Notation . . . . . . . . . . . . . . . . . . . . 255

1 Highlights . . . . . . . . . . . . . . . . . . 256

2 Introduction . . . . . . . . . . . . . . . . . . . . . 257

3 A reminder on flat modules . . . . . . . . . . . . . . . . . . . . . 269

4 An $\aleph_{1}$-perfect set of $\aleph_{1}$-small objects . . . . . . . . . . . . . . . . . . . . . 271

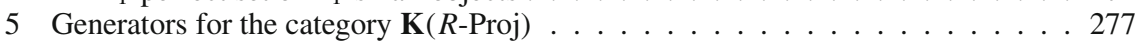

6 Homotopy colimits in $\mathbf{K}(R$-Mod $) \ldots \ldots \ldots \ldots$

7 The compact objects of $\mathbf{K}(R$-Proj $) \ldots \ldots \ldots . \ldots \ldots$

8 The right adjoint to $j_{!}: \mathbf{K}(R$-Proj $) \longrightarrow \mathbf{K}(R$-Flat $) \ldots \ldots . \ldots . \ldots . \ldots 293$

9 The behavior of $\mathbf{K}(R \text {-Proj })^{\perp}$ under tensor product . . . . . . . . . . . . . . 302

Appendix A. When right adjoints respect coproducts . . . . . . . . . . . . 305

References . . . . . . . . . . . . . . . . . . . . . . . 308

\section{Notation}

Let us begin by fixing our notation. All rings $R$ will be assumed associative, with unit.We will have a preference for the left. This means that when we

^ The research was partly supported by the Australian Research Council. 
say " $R$-module", without an adjective, we mean left $R$-module. Similarly "Noetherian" will mean left Noetherian, "coherent" will mean left coherent, and so on. When we wish to refer to a right module, or a right coherent ring, we will use the full names.

Given a ring $R$, we will have occasion to consider several additive categories. All of them will be full subcategories of the category of all (left) $R$-modules. The notation is

$$
\begin{aligned}
R \text {-Mod } & =\text { All (left) } R \text {-modules } \\
R \text {-Proj } & =\text { Projective } R \text {-modules } \\
R \text {-Flat } & =\text { Flat } R \text {-modules } \\
R \text {-Inj } & =\text { Injective } R \text {-modules } \\
R \text {-mod } & =\text { Finitely presented } R \text {-modules } \\
R \text {-proj } & =\text { Finitely generated, projective } R \text {-modules. }
\end{aligned}
$$

Suppose we are given an additive category $\mathcal{A}$; remember, in this article $\mathcal{A}$ will usually be one of $R$-Mod, $R$-Proj, $R$-Flat, $R$-Inj, $R$-mod or $R$-proj. The category $\mathbf{K}(\mathcal{A})$ is the homotopy category of chain complexes in $\mathcal{A}$. The objects are chain complexes

$$
\cdots \longrightarrow X^{i-1} \longrightarrow X^{i} \longrightarrow X^{i+1} \longrightarrow \cdots
$$

and the morphisms are homotopy equivalence classes of chain maps. The derived category $\mathbf{D}(\mathcal{A})$ has the same objects as $\mathbf{K}(\mathcal{A})$, but the morphisms are different. $\operatorname{In} \mathbf{D}(\mathcal{A})$ we formally invert the homology isomorphisms. We will also consider bounded versions; thus $\mathbf{D}^{b}(\mathcal{A})$ is the derived category of bounded complexes in $\mathcal{A}$.

Very occasionally, we will also need to look at the categories $\mathbf{C}(\mathcal{A})$. The objects are the same as in $\mathbf{K}(\mathcal{A})$ and $\mathbf{D}(\mathcal{A})$, but the morphisms are genuine chain maps, not homotopy equivalence classes.

Finally, whenever we deal with a 2-category we will use plain arrows $a \longrightarrow b$ for the 1-morphisms, and double arrows $f \Longrightarrow g$ for the 2-morphisms. If the 2-category is the category of categories, this means that $F$ : $a \longrightarrow b$ will be a functor while $\Phi: F \Longrightarrow G$ will stand for a natural transformation. If the 2-category happens to be the $\mathbf{C}(\mathcal{A})$ of the previous paragraph, that is the objects are chain complexes in $\mathcal{A}$, then $F: a \longrightarrow b$ means a chain map while $\Phi: F \Longrightarrow G$ indicates a chain homotopy.

\section{Highlights}

There are two main theorems in this article. They are:

Theorem 1.1. The homotopy category $\mathbf{K}\left(R\right.$-Proj) is always $\aleph_{1}$-compactly generated, and as a consequence satisfies Brown representability. But it need not be compactly generated. Precisely

(i) If $R$ is right coherent then $\mathbf{K}(R$-Proj) is compactly generated.

(ii) We give an example of an $R$ for which $\mathbf{K}(R$-Proj) is not compactly generated. 
Theorem 1.2. The homotopy category $\mathbf{K}(R$-Proj) has another description. It is equivalent to a Verdier quotient of $\mathbf{K}(R$-Flat) by some subcategory $s \subset \mathbf{K}(R$-Flat). The reader can find six characterizations of the objects of 8 in Facts 2.14 below.

The remarkable fact, which was proved recently in Murfet's thesis, is that the alternative description of $\mathbf{K}(R$-Proj) given in Theorem 1.2 can be extended to non-affine schemes, even to ones in which there are no non-zero projectives. In the next section we will give a much fuller introduction, outlining further results and explaining the relevance to Grothendieck duality.

Acknowledgements: I would like to thank my student Daniel Murfet, as well as two anonymous referees, for many suggestions of improvements on earlier versions.

\section{Introduction}

Grothendieck's duality theory has several aspects, one of which will particularly concern us here. Suppose that $R$ is a ring; a dualizing complex for $R$ is a complex $Y$ of $R-R$ bimodules, so that the functor

$$
X \mapsto \mathcal{R} \mathscr{H o m}(X, Y)
$$

gives an equivalence between the bounded derived category of finitely presented, right $R$-modules, and the dual of the bounded derived category of finitely presented, left $R$-modules. In symbols, the functor

$$
\mathcal{R} \mathscr{H o m}(-, Y): \mathbf{D}^{b}\left(R^{\mathrm{op}}-\mathrm{mod}\right) \longrightarrow \mathbf{D}^{b}(R \text {-mod })^{\mathrm{op}}
$$

is an equivalence. The study of dualizing complexes is interesting for many reasons; historically dualizing complexes formed the backbone of Grothendieck duality, and the theorems were all proved from the properties of dualizing complexes.

Duality theory goes on to deal with schemes which are not affine, and to treat relative versions, where more than one ring (or scheme) is involved. In this article we will largely stick to the simple case. For us, the key feature of a dualizing complex is that it gives an equivalence of triangulated categories

$$
\mathbf{D}^{b}\left(R^{\mathrm{op}} \text {-mod }\right){ }^{\mathrm{op}} \cong \mathbf{D}^{b}(R \text {-mod }) .
$$

Over the years there has developed a tremendous body of literature in this field. Many people have written beautiful papers, providing deep insights; too many for us to list here. What I want to report here is a novel, new angle. In the last couple of years there have been several results, which allow us to approach this old subject in a totally new way. Because this is all so new, and not widely known, I felt it appropriate to summarize the three articles in which the results appeared. This makes the introduction unusually long; approximately half of it is spent discussing results which have recently appeared elsewhere. I hope the reader will bear with me. 
To state the new results, let me first remind the reader of the general formalism of compact objects in a [TR5] triangulated category. We recall some basic definitions.

Definition 2.1. A triangulated category satisfies [TR5] if it has arbitrary small coproducts.

Example 2.2. The following are examples of triangulated categories satisfying [TR5]:

(i) The category $\mathbf{K}(R$-Proj), for any ring $R$.

(ii) The category $\mathbf{K}(R$-Inj), as long as $R$ is Noetherian. The point is that, over a Noetherian ring, direct sums of injective modules are injective.

Definition 2.3. Let $\mathcal{T}$ be a triangulated category satisfying [TR5]. An object $k \in \mathcal{T}$ is compact if any map

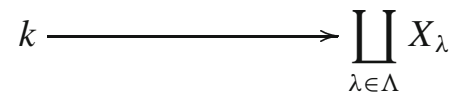

factors as

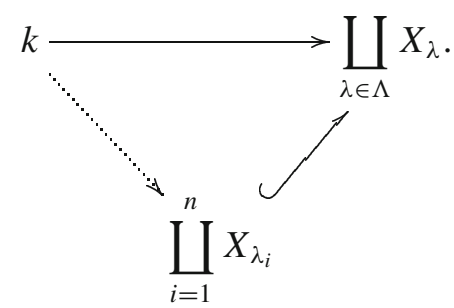

That is any map, from $k$ into an arbitrary coproduct, factors through a finite coproduct.

Definition 2.4. Let $\mathcal{T}$ be a triangulated category satisfying [TR5]. The full subcategory of all compact objects in $\mathcal{T}$ will be denoted $\mathcal{T}^{c}$.

Definition 2.5. Let $\mathcal{T}$ be a triangulated category satisfying [TR5]. We say that $\mathcal{T}$ is compactly generated if

(i) $\mathcal{T}^{c}$ is essentially small.

(ii) $\mathcal{T}^{c}$ generates $\mathcal{T}$. This means that one of the two equivalent conditions below holds:

(a) If $\&$ is a triangulated subcategory of $\mathcal{T}$, closed under coproducts and containing $\mathcal{T}^{c}$, then $s=\mathcal{T}$.

(b) If $X \in \mathcal{T}$ is any non-zero object, then there is a non-zero map $k \longrightarrow X$ with $k \in \mathcal{T}^{c}$.

This reminds us of the basic definitions in the theory of compactly generated triangulated categories. The relation of dualizing complexes, with compactly generated triangulated categories, comes from two recent theorems, which appeared in a couple of beautiful articles in 2005: 
Theorem 2.6 (Krause [9]). Suppose that $R$ is a Noetherian ring. ${ }^{1}$ Then the category $\mathbf{K}(R$-Inj) is compactly generated. Furthermore, there is a natural equivalence

$$
\mathbf{K}(R-\operatorname{Inj})^{c} \cong \mathbf{D}^{b}(R \text {-mod }) .
$$

Theorem 2.7 (Jørgensen [8]). Let $R$ be a sufficiently nice ring. Then the category $\mathbf{K}(R$-Proj) is compactly generated. Furthermore, there is a natural equivalence

$$
\mathbf{K}(R-\operatorname{Proj})^{c} \cong \mathbf{D}^{b}\left(R^{\mathrm{op}}-\mathrm{mod}\right){ }^{\mathrm{op}} .
$$

We will come to the precise hypotheses, which Jørgensen [8, Theorem 2.4] places on the ring $R$, later in the introduction, when we discuss our improvements to the result. It turns out that Jørgensen's theorem is not best possible. For now, it is simplest to leave vague the precise hypotheses on $R$.

Suppose $R$ is a sufficiently nice notherian ring, so that Theorems 2.6 and 2.7 both apply. Suppose $Y$ is a dualizing complex. We have a diagram

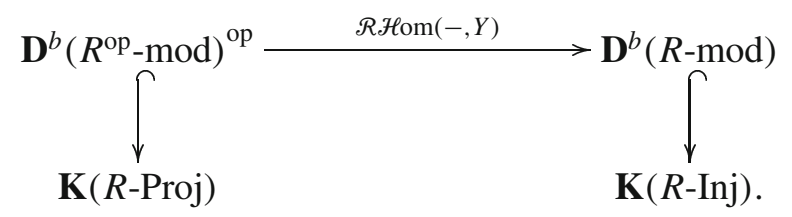

Recall: the dualizing complex $Y$ gives an equivalence $\mathcal{R} \mathscr{H}$ om $(-, Y)$ from $\mathbf{D}^{b}\left(R^{\mathrm{op}} \text {-mod }\right)^{\mathrm{op}}$ to $\mathbf{D}^{b}(R$-mod $)$, Theorem 2.7 embeds $\mathbf{D}^{b}\left(R^{\mathrm{op}}-\text { mod }\right)^{\mathrm{op}}$ as the compact objects in $\mathbf{K}\left(R\right.$-Proj), while Theorem 2.6 embeds $\mathbf{D}^{b}(R$-mod) as the compact objects in $\mathbf{K}(R$-Inj). It is natural to ask whether the equivalence of categories $\mathbf{D}^{b}\left(R^{\mathrm{op}} \text {-mod }\right)^{\text {op }} \longrightarrow \mathbf{D}^{b}(R$-mod $)$ can be extended to an equivalence $\mathbf{K}(R$-Proj $) \longrightarrow \mathbf{K}(R$-Inj).

This question was addressed by a recent article of Iyengar and Krause [7]. Replacing $Y$ by an injective resolution, we may assume that $Y$ is a complex of $R-R$ bimodules, injective both as right and as left $R$-modules. The authors noted first that the square

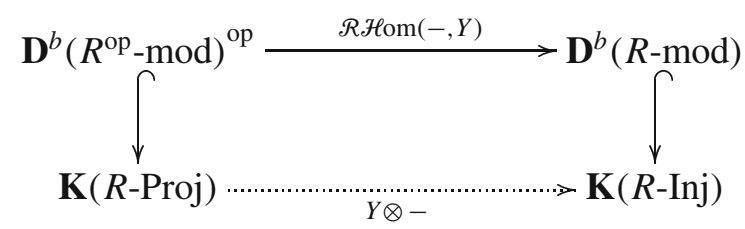

commutes; taking $X$ to $Y \otimes X$ defines a functor $\mathbf{K}(R$-Proj $) \longrightarrow \mathbf{K}(R$-Inj), which restricts on compact objects to the equivalence $\mathcal{R} \mathcal{H o m}(-, Y)$ : $\mathbf{D}^{b}\left(R^{\mathrm{op}}-\mathrm{mod}\right)^{\mathrm{op}} \longrightarrow \mathbf{D}^{b}(R$-mod $)$. At this point the theory of compactly gen-

${ }^{1}$ Krause's result is more general than what we state here. We give the narrow version, valid for rings. 
erated triangulated categories kicks in. The functor $Y \otimes-: \mathbf{K}(R$-Proj $) \longrightarrow$ $\mathbf{K}(R$-Inj) is a triangulated functor respecting coproducts, and inducing an equivalence on the subcategories of compact objects. Formal nonsense guarantees that $Y \otimes-: \mathbf{K}(R$-Proj $) \longrightarrow \mathbf{K}(R$-Inj $)$ must be an equivalence of categories.

Let us consider the following properties, which a ring $R$ might possess:

(i) There is a dualizing complex for $R$.

(ii) There is an equivalence of triangulated categories $\mathbf{D}^{b}\left(R^{\mathrm{op}}-\mathrm{mod}\right)^{\mathrm{op}} \cong$ $\mathbf{D}^{b}(R$-mod).

(iii) There is an equivalence of triangulated categories $\mathbf{K}(R$-Proj $) \cong$ $\mathbf{K}(R$-Inj).

Recall that we are assuming that $R$ is a sufficiently nice ring, so that Theorems 2.6 and 2.7 both apply. The observation we explained above, due to Iyengar and Krause, is that (i) $\Longrightarrow$ (iii). The fact that (iii) $\Longrightarrow$ (ii) is trivial; an equivalence $\mathbf{K}(R$-Proj $) \longrightarrow \mathbf{K}(R$-Inj) must induce an equivalence on the compact objects. That is, for sufficiently nice rings $R$ we have the implications

$$
\text { (i) } \Longrightarrow \text { (iii) } \Longrightarrow \text { (ii). }
$$

It is not known, for a general $R$, whether (ii) $\Longrightarrow$ (i). However, two facts are known

(1) If the ring $R$ is commutative, Noetherian and local, then (ii) $\Longrightarrow$ (i).

(2) There are examples of commutative, Noetherian, local rings where (i) fails.

This means that, in the extremely special situation where we know that (i), (ii) and (iii) are equivalent, it is possible for all three to fail. The conditions are subtle. In the past, Grothendieck duality has been studied by means of (i) and (ii). Condition (iii) is very new, and so far our understanding is sketchy. Nevertheless, the major thrust of this article is to try to persuade the reader that (iii) offers a very promising new angle on this old problem.

Let me return to the next relevant result in the paper of Iyengar and Krause. The equivalence of categories

$$
Y \otimes-: \mathbf{K}(R \text {-Proj }) \longrightarrow \mathbf{K}(R \text {-Inj })
$$

must have a quasi-inverse, and the paper [7] goes on to study it. First the authors observe that the functor $Y \otimes-: \mathbf{K}(R$-Proj $) \longrightarrow \mathbf{K}(R$-Inj $)$ can be factored as

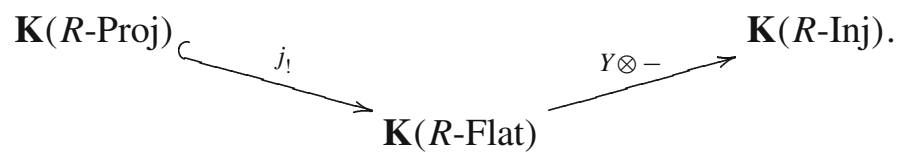


In this factorization, the map $j_{1}$ is the obvious inclusion, and the functor $Y \otimes-$ is the natural functor, taking $X$ to $Y \otimes X$; the point is that this functor extends from $\mathbf{K}$ ( $R$-Proj) to the larger $\mathbf{K}(R$-Flat). The inclusion

$$
j_{!}: \mathbf{K}(R \text {-Proj }) \longrightarrow \mathbf{K}(R \text {-Flat })
$$

respects coproducts. Since $R$ is assumed sufficiently nice, Jørgensen's Theorem 2.7 tells us that the category $\mathbf{K}(R$-Proj $)$ is compactly generated. The general theory of compactly generated triangulated categories comes to our aid again: the coproduct-preserving functor $j_{!}: \mathbf{K}(R$-Proj $) \longrightarrow \mathbf{K}(R$-Flat $)$, whose domain is compactly generated, must have a right adjoint $j^{*}$. Also, the functor

$$
Y \otimes-: \mathbf{K}(R \text {-Flat }) \longrightarrow \mathbf{K}(R \text {-Inj })
$$

clearly has a right adjoint; the right adjoint is the functor taking $X \in$ $\mathbf{K}(R$-Inj) to

$$
\mathscr{H o m}(Y, X) \text {. }
$$

We deduce a diagram of adjoints

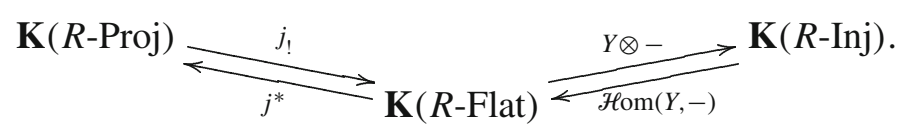

In Diagram (1), every arrow is left adjoint to the one below it. The composite $\mathbf{K}(R$-Proj $) \longrightarrow \mathbf{K}(R$-Inj $)$ is an equivalence of categories, and its right adjoint $j^{*} \mathscr{H} \operatorname{om}(Y,-)$ must be a quasi-inverse.

Let us pause for a second. Grothendieck duality deals with schemes, not all of which are affine. For a general scheme $X$ the theory looks at dualizing complexes, which give equivalences of categories

$$
\mathbf{D}^{b}(\mathrm{Coh} / X)^{\mathrm{op}} \cong \mathbf{D}^{b}(\mathrm{Coh} / X) \text {. }
$$

If $X=\operatorname{Spec}(R)$ is affine, then Theorems 2.6 and 2.7 permitted us to extend this to an equivalence $\mathbf{K}(R$-Proj $) \longrightarrow \mathbf{K}(R$-Inj). From the perspective of Grothendieck duality these facts might be very nice, but they seem of limited value; on the face of it they can only be used when $X$ is affine. The point is that the category $\mathbf{K}(R$-Proj) is nearly worthless on general schemes. For a general scheme $X$, there are very few projectives in the category of quasi-coherent sheaves on $X$.

The results of Iyengar and Krause had the potential of changing this. Diagram (1) embeds the category $\mathbf{K}(R$-Proj $) \cong \mathbf{K}(R$-Inj $)$, in two different ways, into the category $\mathbf{K}(R$-Flat $)$. Note that, in Diagram (1), the functors $j_{!}$and $\mathscr{H} \operatorname{om}(Y,-)$ must both be fully faithful. Since the category $\mathbf{K}(R$-Flat $)$ can be generalized sensibly to any scheme $Y$, I wanted to understand the two embeddings better. A thorough understanding of the affine case often leads to the right global generalization. 
In this article I will present the results only in the context of affine schemes $\operatorname{Spec}(R)$. As a result of the $\mathrm{PhD}$ thesis of Daniel Murfet we now know that the results obtained in the affine case, in this paper and its sequel, do in fact naturally lead to global generalizations, valid for any sufficiently nice scheme. I will occasionally give glimpses of Murfet's results, to indicate what may be found in his upcoming thesis. But, aside from these occasional snippets from Murfet's work, I stick entirely to affine schemes. In developing the affine case I tried to be as general as possible, and, in particular, I tried not to assume any commutativity hypothesis. In recent years some of the most exciting developments in Grothendieck duality have been in the area of non-commutative geometry, and from the results of this paper it is possible to formulate some conjectures. Although some of the questions are obvious I will say little about them here; a discussion will appear elsewhere.

We would like to develop a thorough understanding of Diagram (1), to allow us to generalize to the non-affine case. We have no problem giving global analogs of flat modules and injective modules; the part of the diagram that begs a better understanding is

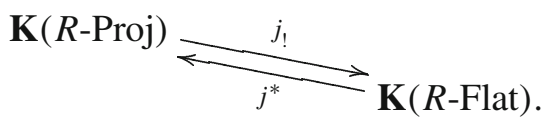

After all, it is the projectives that fail to make sense globally. In this article we will try to better understand this pair of functors, and its relation with the right hand side of Diagram (1).

Note that what we are really given, absolutely for free, is the inclusion

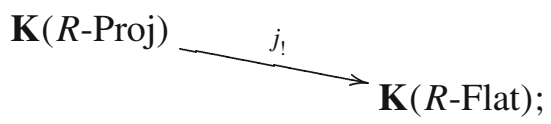

the projective modules always include into the flat modules. This inclusion always preserves coproducts. To produce the right adjoint $j^{*}$ we appealed to Brown representability; Jørgensen's Theorem 2.7 tells us that, so long as the ring $R$ is "sufficiently nice", $\mathbf{K}(R$-Proj $)$ is compactly generated and therefore satisfies Brown representability. As I have already explained, I wanted to develop the theory with a minimum of assumptions on the ring $R$. The first order of business was therefore to try to obtain an optimal version of Jørgensen's theorem. To state our improvements of Jørgensen's theorem it is best to work in the framework of well generated triangulated categories. For the next few facts we state, we assume the reader has some basic familiarity with the notation of [11]. What we prove is

Facts 2.8. The following holds for the triangulated category $\mathbf{K}(R$-Proj):

(i) For every ring $R$, the category $\mathbf{K}\left(R\right.$-Proj) is an $\aleph_{1}$-compactly generated triangulated category. We will produce an explicit $\aleph_{1}$-perfect set of $\aleph_{1}$-small generators. An immediate consequence is that $\mathbf{K}(R$-Proj) 
is well generated; a triangulated category, with coproducts, is well generated if and only if it has an $\alpha$-perfect set of $\alpha$-small generators, for some regular cardinal $\alpha$. Such a set is called an $\alpha$-compact generating set. In our case $\alpha=\aleph_{1}$ works. The reader is referred to Remark 2.9 and to Construction 4.3 for the explicit definition of the set $T$ of generators, and to Theorem 5.9 where we complete the proof that it is an $\aleph_{1}$-compact generating set.

(ii) We give a complete description of the categories $\{\mathbf{K}(R \text {-Proj })\}^{\alpha}$, for every regular cardinal $\alpha$. That is we tell the reader precisely, for any ring $R$ and any regular cardinal $\alpha$, what are the $\alpha$-compact objects. The $\aleph_{0}$-compact objects are what, in the usual terminology, would be called just the compact objects of $\mathbf{K}(R$-Proj). For the description of $\{\mathbf{K}(R \text {-Proj })\}^{\aleph_{1}}$ see Proposition 7.4, the description of $\{\mathbf{K}(R \text {-Proj })\}^{\aleph_{0}}$ may be found in Proposition 7.12, and for other $\alpha$ see Proposition 7.5.

(iii) We show that, if the ring $R^{\text {op }}$ is a coherent ring, ${ }^{2}$ then the category $\mathbf{K}(R$-Proj) is compactly generated. For the proof see Proposition 7.14. We remind the reader: compactly generated is a special case of well generated. It is an $\alpha$-compactly generated triangulated category, in the special case where $\alpha=\aleph_{0}$.

(iv) We produce an example of a ring $R$, where the category $\mathbf{K}(R$-Proj) is not compactly generated; see Example 7.16. Needless to say, $R^{\text {op }}$ is not coherent.

Remark 2.9. In Fact 2.8(i) we promised the reader an explicit set $T$ of generators for the category $\mathbf{K}(R$-Proj). Needless to say there are many choices. The generators we will find convenient, in this article, are the objects of $\mathbf{K}^{+}(R$-proj); these are the bounded below complexes of finitely generated, projective $R$-modules. Up to isomorphism there is a set of such objects.

The slight wrinkle is that we will find it convenient to choose a set $T$ of representatives for the isomorphism classes in the category $\mathbf{C}^{+}(R$-proj); that is, we will choose representatives of chain complexes up to isomorphism, not up to homotopy equivalence. This means that there is a great deal of redundancy in the set $T$. Many pairs of objects of $T$ are isomorphic in $\mathbf{K}(R$-Proj).

Remark 2.10. We should tell the reader how the results listed in Fact 2.8 compare to what may be found in the literature. What was known, up to now, were the clever results of Jørgensen [8, §2 and §3]; we already mentioned them, albeit a little vaguely. Until now, all we have said is that Jørgensen assumes that $R$ is a "sufficiently nice ring"; since we are about to explain how our results improve on Jørgensen's, it is time to give the hypotheses of his theorem in detail. The precise statement that Jørgensen

2 We remind the reader that a ring is coherent if every finitely generated ideal is finitely presented. All Noetherian rings are coherent. To say that $R^{\mathrm{op}}$ is (left) coherent means that $R$ is right coherent. 
proved, in [8, Theorem 2.4], was that, as long as the ring $R$ is both left and right coherent, and provided every flat left $R$-module has finite projective dimension, then the category $\mathbf{K}(R$-Proj $)$ is compactly generated. Fact 2.8(iii) improves on this by removing the hypothesis that flat modules have finite projective dimension, and by requiring $R$ only to be right coherent. Jørgensen also computed for us the compact objects in $\mathbf{K}(R$-Proj), in the special case where $R$ is one of the rings for which he could prove $\mathbf{K}(R$-Proj) compactly generated. We already mentioned this; he identified the compact objects with $\mathbf{D}^{b}\left(R^{\mathrm{op}}-\mathrm{Proj}\right)^{\mathrm{op}}$. See [8, Theorem 3.2].

We can show the compact generation of $\mathbf{K}(R$-Proj) for $R$ more general than in Jørgensen [8, Theorem 2.4], we can exhibit an $R$ for which $\mathbf{K}(R$-Proj) is not compactly generated, and, for every $R$, we can compute the subcategory $\{\mathbf{K}(R \text {-Proj })\}^{c}=\{\mathbf{K}(R \text {-Proj })\}^{\aleph_{0}}$, that is the category of compact objects. Jørgensen only computes $\{\mathbf{K}(R \text {-Proj })\}^{c}$ if $R$ is sufficiently nice.

Beside this, we also give an analysis of the category $\{\mathbf{K}(R \text {-Proj })\}^{\alpha}$ for larger regular cardinals $\alpha$. Especially relevant is $\{\mathbf{K}(R \text {-Proj })\}^{\aleph_{1}}$; it is useful since the category $\mathbf{K}\left(R\right.$-Proj) is always $\aleph_{1}$-compactly generated, but not necessarily $\aleph_{0}$-compactly generated. It is natural to work with the smallest $\alpha$ for which the category is $\alpha$-compactly generated; for a general $\mathbf{K}(R$-Proj), this minimal $\alpha$ happens to be $\aleph_{1}$.

Remark 2.11. For the applications in this article, the most important is Fact 2.8(i). It tells us that, for any ring $R$, the category $\mathbf{K}(R$-Proj) is well generated, and hence satisfies Brown representability. The inclusion $j_{1}: \mathbf{K}(R$-Proj $) \longrightarrow \mathbf{K}(R$-Flat $)$ respects coproducts, and Brown representability now informs us that it must have a right adjoint. In other words the adjoint pair of functors

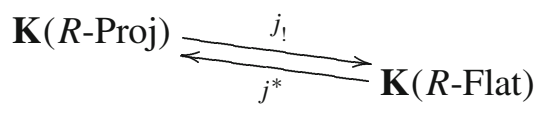

always exists, with no hypothesis on the ring $R$.

Remark 2.12. The functor $j_{1}$ is fully faithful, and has a right adjoint $j^{*}$. Formal nonsense tells us that the adjoint, right or left, of a fully faithful, triangulated functor is always a Verdier quotient. The same formal nonsense also tells us that the adjoint, right or left, of a Verdier quotient is always fully faithful. Let us remind the reader of these statements, a little more precisely. Suppose we are given a fully faithful triangulated functor $j_{!}: \delta \longrightarrow \mathcal{T}$, with a right adjoint $j^{*}: \mathcal{T} \longrightarrow \&$. What do we mean by saying that $j^{*}$ "is" a Verdier quotient?

It is natural to look at the kernel of the functor $j^{*}$; that is, let $s^{\perp} \subset \mathcal{T}$ be the full subcategory of all objects annihilated by the functor $j^{*}$. It is not difficult to see that the kernel $s^{\perp}$ can also be described by the formula

$$
\mathrm{ob}\left(8^{\perp}\right)=\left\{y \in \mathcal{T} \mid \operatorname{Hom}\left(j_{!} s, y\right)=0 \forall s \in \delta\right\} ;
$$


this explains the notation, $\delta^{\perp}$ is orthogonal to $\&$ with respect to the Hompairing. Let $i_{*}: \delta^{\perp} \longrightarrow \mathcal{T}$ be the inclusion. We would like to say that the functor $j^{*}$ identifies with the Verdier quotient map $\pi: \mathcal{T} \longrightarrow \mathcal{T} / \mathcal{S}^{\perp}$. The precise assertion is that, in the diagram below

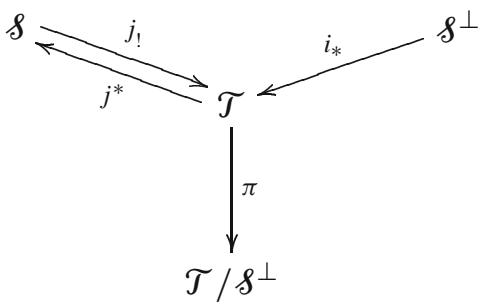

we have

(i) The composite $\pi j_{!}: \& \longrightarrow \mathcal{T} / \mathcal{S}^{\perp}$ is an equivalence of categories.

(ii) The composite $\pi j_{1} j^{*}: \mathcal{T} \longrightarrow \mathcal{T} / \mathcal{S}^{\perp}$ is naturally isomorphic to $\pi$ : $\mathcal{T} \longrightarrow \mathcal{T} / \mathcal{S}^{\perp}$. More precisely we have a counit of adjunction $\varepsilon:$ $j_{!} j^{*} \Longrightarrow 1$, and hence a natural transformation $\pi \varepsilon: \pi j_{!} j^{*} \Longrightarrow \pi$. We assert that $\pi \varepsilon$ is an isomorphism.

From (i) we learn that the equivalence of categories $\pi j_{!}: \& \longrightarrow \mathcal{T} / \mathcal{s}^{\perp}$ permits us to identify the two categories $\delta$ and $\mathcal{T} / \delta^{\perp}$. And (ii) teaches us that, up to the natural isomorphism $\pi \varepsilon$, the triangle

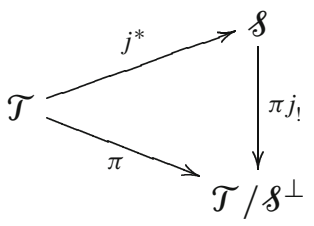

commutes. If we identify $\delta$ and $\mathcal{T} / \delta^{\perp}$ via the equivalence $\pi j_{\text {! }}$ then, up to the natural isomorphism $\pi \varepsilon$, the map $j^{*}: \mathcal{T} \longrightarrow \delta$ is identified with the Verdier quotient map $\pi: \mathcal{T} \longrightarrow \mathcal{T} / \mathcal{s}^{\perp}$. This is a more precise version of the crude assertion that $j^{*}$ "is" a Verdier quotient map.

Remark 2.13. These formal facts, about adjoints of fully faithful functors and Verdier quotients, apply more generally, to categories that need not be triangulated; see [6, I.1.3]. The triangulated version may be found in [12, Proposition 2.3.3, especially Parts (g) and (e)]. The results were rediscovered later by Bousfield, who applied them extensively in the homotopy category. See [4] for the rediscovery of the results, and many subsequent articles by Bousfield, and by other homotopy theorists, for applications. In this article we will give references to the more modern [11, Chap. 9], mostly because the notation is more compatible with the one used here. The reader should be warned that the notation here is close, but not identical, with [11, Chap. 9]; the difference is that the perpendiculars are on opposite sides. What we write as $\delta^{\perp}$ here would be denoted ${ }^{\perp} \delta$ there. 
In our special case, where $\delta=\mathbf{K}(R$-Proj $)$ and $\mathcal{T}=\mathbf{K}(R$-Flat $)$, we deduce a diagram

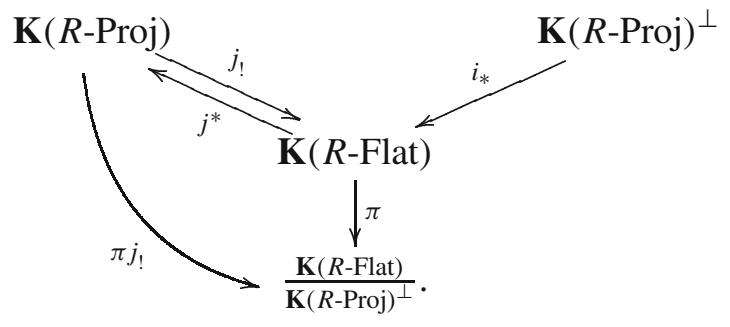

The existence of this diagram comes for free, but to make use of it we need to understand the subcategory $\mathbf{K}(R \text {-Proj })^{\perp} \subset \mathbf{K}(R$-Flat $)$; in the language of homotopy theory, we need to understand the $\mathbf{K}(R$-Proj)-local objects in $\mathbf{K}(R$-Flat). The following results give a string of characterizations:

Facts 2.14. Let $R$ be an arbitrary ring. Let $X$ be an object of $\mathbf{K}(R$-Flat). Then the following are equivalent:

(i) $X$ lies in the subcategory $\mathbf{K}(R \text {-Proj })^{\perp} \subset \mathbf{K}(R$-Flat $)$.

(ii) $X$ is a filtered direct limit of contractible complexes of projective $R$-modules.

(iii) $X$ is acyclic, and, for every integer $i \in \mathbb{Z}$, the image $I^{i}$ of the differential $\partial^{i}: X^{i} \longrightarrow X^{i+1}$ is a flat $R$-module.

(iv) If $Z$ is any chain complex of right $R$-modules, then $Z \otimes_{R} X$ is acyclic.

(v) For every right $R$-module $M$, the complex $M \otimes_{R} X$ is acyclic.

(vi) For every finitely presented left module $N$, the complex $\mathcal{H o m}_{R}(N, X)$ is acyclic.

The proof that (i) $\Longrightarrow$ (ii) $\Longrightarrow$ (iii) $\Longrightarrow$ (i) may be found in Theorem 8.6. The proof that (ii) $\Longrightarrow$ (iv) $\Longrightarrow$ (v) $\Longrightarrow$ (vi) $\Longrightarrow$ (iii) may be found in Corollary 9.4. It should be noted that the equivalences (ii) $\Longleftrightarrow$ (iii) $\Longleftrightarrow$ (iv) $\Longleftrightarrow$ (v) $\Longleftrightarrow$ (vi) are quite easy; the work is in proving (i) $\Longrightarrow$ (ii) and (iii) $\Longrightarrow$ (i).

Remark 2.15. To illustrate the non-triviality of the implication (iii) $\Longrightarrow$ (i) let us note a curious aside. Suppose $X$ is an acyclic chain complex

$$
\stackrel{\partial^{i-2}}{\longrightarrow} X^{i-1} \stackrel{\partial^{i-1}}{\longrightarrow} X^{i} \stackrel{\partial^{i}}{\longrightarrow} X^{i+1} \stackrel{\partial^{i+1}}{\longrightarrow}
$$

of projective modules. Suppose that, for each $i \in \mathbb{Z}$, the image $I^{i}$ of the differential $\partial^{i}: X^{i} \longrightarrow X^{i+1}$ is a flat $R$-module.

By definition $X$ belongs to $\mathbf{K}(R$-Proj), and by (iii) $\Longrightarrow$ (i) $X$ also belongs to $\mathbf{K}(R \text {-Proj })^{\perp}$. Hence $X$ must be null homotopic. The module $I^{i}$, being a direct summand of $X^{i}$, is forced to be projective. What is curious about this aside is that the statement is the assertion that certain flat modules have to be projective; it does not mention triangulated categories. I do not know an elementary proof, a proof which avoids homotopy categories. 
Remark 2.16. I mentioned that the motivating reason, for the study undertaken in this article, was to see if there is a way to generalize to non-affine schemes the new approach to Grothendieck duality. The problem, as we explained, is that the category $\mathbf{K}(R$-Proj $)$ is not very reasonable except in the affine case. In Remark 2.12 we noted that the functor $j^{*}: \mathbf{K}(R$-Flat $) \longrightarrow$ $\mathbf{K}(R$-Proj) can be identified, up to natural isomorphism, with the functor $\pi$ : $\mathbf{K}(R$-Flat $) \longrightarrow \mathbf{K}(R$-Flat $) / \mathbf{K}(R \text {-Proj })^{\perp}$. And the many characterizations of $\mathbf{K}(R \text {-Proj })^{\perp}$, given in Facts 2.14 , permit us to generalize $\pi$ to arbitrary schemes. The question I asked Murfet, which led to his $\mathrm{PhD}$ thesis, was whether the characterization of $\mathbf{K}(R \text {-Proj })^{\perp}$ given in Fact 2.14(iii) generalizes well to arbitrary schemes. The answer is Yes, as Murfet's thesis shows.

We return to this article, and the world of affine schemes $\operatorname{Spec}(R)$. Until now $R$ was arbitrary. From now let us assume that $R$ is a Noetherian ring, and let $Y$ be a complex of $R-R$ bimodules, injective as left $R$-modules. Then tensoring with $Y$ defines a functor

$$
Y \otimes-: \mathbf{K}(R \text {-Flat }) \longrightarrow \mathbf{K}(R \text {-Inj }) .
$$

Facts 2.17. Let the hypotheses be as above. Then the functor

$$
Y \otimes-: \mathbf{K}(R \text {-Flat }) \longrightarrow \mathbf{K}(R \text {-Inj })
$$

takes any object $X \in \mathbf{K}(R \text {-Proj })^{\perp}$ to a contractible complex $Y \otimes X$. See Corollary 9.7(ii) for the proof.

That is, the functor $Y \otimes-: \mathbf{K}(R$-Flat $) \longrightarrow \mathbf{K}(R$-Inj) factors through the quotient category $\mathbf{K}(R$-Flat $) / \mathbf{K}(R \text {-Proj })^{\perp}$. In the diagram below

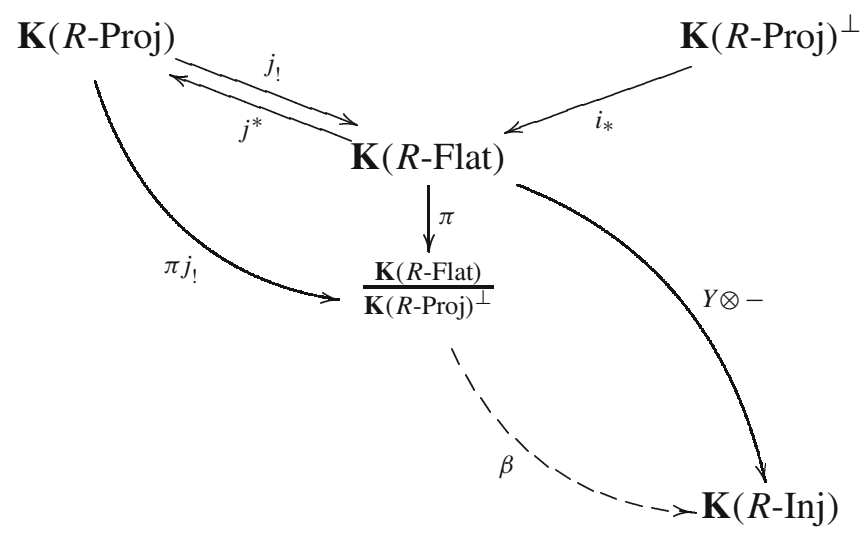

we can find a functor $\beta$, so that the functor $Y \otimes-$ may be factored as $\beta \pi$. The commutativity allows us to compute that, up to natural isomorphism,

$$
\begin{aligned}
\{Y \otimes-\} j_{!} j^{*} & =\beta \pi j_{!} j^{*} \\
& \cong \beta \pi \\
& =\{Y \otimes-\} .
\end{aligned}
$$


This is true for any complex $Y$ of $R-R$ bimodules, injective as left $R$-modules. If $Y$ happens to be a dualizing complex, then the theorem of Iyengar and Krause tells us that the composite $\{Y \otimes-\} j_{!}: \mathbf{K}(R$-Proj $) \longrightarrow \mathbf{K}(R$-Inj $)$ is an equivalence of categories. The isomorphism

$$
\left[\{Y \otimes-\} j_{!}\right] \circ j^{*} \cong\{Y \otimes-\}
$$

tells us that the functor $j^{*}$ may be identified, up to equivalence, with the functor $Y \otimes-$.

Now look back at Diagram (1) on p. 261. The functor $Y \otimes-$ has a right adjoint $\mathscr{H}$ om $(Y,-)$; this forces the functor $j^{*}$ to have a right adjoint $j_{*}$. If $R$ is a sufficiently nice ring, and if a dualizing complex exists for $R$, we therefore know that there are functors

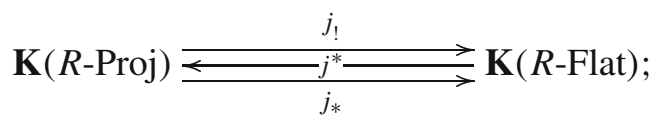

that is, the functor $j^{*}$ has a left and a right adjoint. We also know that the functor $j_{*}$ is related to the dualizing complex $Y$. It is natural to ask whether it is possible to reverse the process; is it possible to prove the existence of the right adjoint $j_{*}$, and deduce facts about the dualizing complex $Y$ ? One of the facts I can prove so far, which will be included in a sequel to this paper, is that, for any ring $R$, the functor $j^{*}$ has a right adjoint. Murfet was able to generalize this to sufficiently nice schemes.

Perhaps we should explain why this is remarkable. We have a unit of adjunction $\eta: 1 \Longrightarrow j_{*} j^{*}$. What $\mathrm{I}$ have just asserted is that it exists almost unconditionally, for every ring and for many schemes. If there is a dualizing complex $Y$, and if the ring (or scheme) is sufficiently nice, then the results of this article identify the functor $j^{*}$ with $Y \otimes-$, while $j_{*}$ is identified with $\mathscr{H} \operatorname{om}(Y,-)$; this makes the unit of adjunction quite explicit. For any $? \in \mathbf{K}(R$-Flat $)$, the map $\eta_{\text {? }}: ? \longrightarrow j_{*} j^{*}$ ? comes down to

$$
? \stackrel{\eta_{?}}{\longrightarrow} \operatorname{Hom}(Y, Y \otimes ?) .
$$

The curious fact, which I do not yet understand, is that this map can be defined even in the absence of a dualizing complex. Let us specialize to the case $?=R$. If there is a dualizing complex then $j_{*} j^{*} R \cong \mathscr{H}$ om $(Y, Y)$. But even if there is no dualizing complex there is a complex $j_{*} j^{*} R$, of flat $R$-modules, unique up to homotopy equivalence. Even when there is no $Y$, we can compute what $\mathscr{H}$ om $(Y, Y)$ must be.

Remark 2.18. It has been known, for a long time, that the map $R \longrightarrow$ $\mathscr{H} \operatorname{om}(Y, Y)$ must be a quasi-isomorphism, if $Y$ is a dualizing complex. However, $\operatorname{Hom}(Y, Y)$ is almost never homotopy equivalent to $R$. For example, in the case $R=\mathbb{Z}$ we can easily compute that $\mathscr{H o m}(Y, Y)$ is homotopy equivalent to the complex

$$
0 \longrightarrow \mathbb{Q} \oplus \mathbb{A}_{\mathbb{Z}} \longrightarrow \mathbb{A}_{\mathbb{Q}} \longrightarrow 0,
$$


where $\mathbb{A}_{\mathbb{Z}}$ is the ring of integral adeles, that is

$$
\mathbb{A}_{\mathbb{Z}}=\mathbb{R} \oplus \prod_{p \text { prime }} \mathbb{Z}_{p},
$$

while $\mathbb{A}_{\mathbb{Q}}=\mathbb{Q} \otimes \mathbb{A}_{\mathbb{Z}}$ is the ring of rational adeles. Since the only chain map

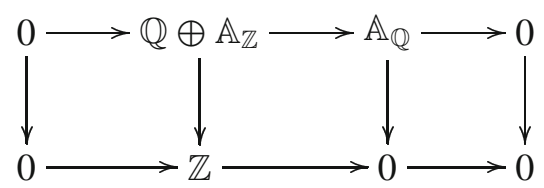

is zero, $\mathscr{H}$ om $(Y, Y)$ cannot be homotopy equivalent to $\mathbb{Z}$. We have learned that there is a construction of the complex $\mathcal{H}$ om $(Y, Y)$ purely in terms of the adjoints $j^{*}$ and $j_{*}$, and the construction generalizes to all rings, even ones with no dualizing complexes.

\section{A reminder on flat modules}

Let $R$ be a ring. We will heavily rely on the fact that any flat $R$-module is a filtered direct limit of projective modules. We will use some standard lemmas about flat modules a great deal; because we rely on them so heavily, we include the (standard) proofs.

Recall the following. Given $R$-modules $M$ and $Z$, and an $R-R$ bimodule $I$, there is a natural map

$$
\varphi: \operatorname{Hom}_{R}(M, I) \otimes_{R} Z \longrightarrow \operatorname{Hom}_{R}\left(M, I \otimes_{R} Z\right) .
$$

For a pair of elements $f \in \operatorname{Hom}_{R}(M, I)$ and $z \in Z$, the map $\varphi$ takes the tensor $f \otimes z \in \operatorname{Hom}_{R}(M, I) \otimes_{R} Z$ to the function

$$
M \stackrel{f}{\longrightarrow} I=I \otimes_{R} R \stackrel{1 \otimes z}{\longrightarrow} I \otimes_{R} Z,
$$

where the function $z: R \longrightarrow Z$ is right multiplication by $z \in Z$. This map $\varphi$ is natural in all three variables, $M, I$ and $Z$. In this section we will use only the naturality in $M$, and hence we label the map $\varphi_{M}$.

Lemma 3.1. With the notation as above, suppose that $M$ is finitely presented and $Z$ is flat. Then the map

$$
\varphi_{M}: \operatorname{Hom}_{R}(M, I) \otimes_{R} Z \longrightarrow \operatorname{Hom}_{R}\left(M, I \otimes_{R} Z\right)
$$

is an isomorphism.

Proof. Choose a finite presentation for $M$; that is, choose finitely generated, free modules $F_{1}$ and $F_{0}$, and an exact sequence

$$
F_{1} \longrightarrow F_{0} \longrightarrow M \longrightarrow 0 .
$$


By the right exactness of $\operatorname{Hom}_{R}(-, I)$, the sequence

$$
0 \longrightarrow \operatorname{Hom}_{R}(M, I) \longrightarrow \operatorname{Hom}_{R}\left(F_{0}, I\right) \longrightarrow \operatorname{Hom}_{R}\left(F_{1}, I\right)
$$

is an exact sequence of right $R$-modules. Because $Z$ is flat, the sequence $0 \rightarrow \operatorname{Hom}_{R}(M, I) \otimes_{R} Z \rightarrow \operatorname{Hom}_{R}\left(F_{0}, I\right) \otimes_{R} Z \rightarrow \operatorname{Hom}_{R}\left(F_{1}, I\right) \otimes_{R} Z$ is also exact. We deduce a commutative diagram with exact rows $0 \rightarrow \operatorname{Hom}_{R}(M, I) \otimes_{R} Z \rightarrow \operatorname{Hom}_{R}\left(F_{0}, I\right) \otimes_{R} Z \rightarrow \operatorname{Hom}_{R}\left(F_{1}, I\right) \otimes_{R} Z$

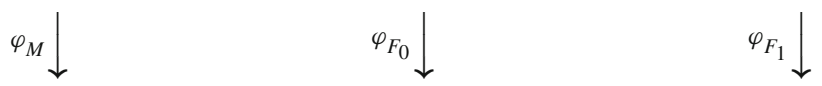

$0 \rightarrow \operatorname{Hom}_{R}\left(M, I \otimes_{R} Z\right) \rightarrow \operatorname{Hom}_{R}\left(F_{0}, I \otimes_{R} Z\right) \rightarrow \operatorname{Hom}_{R}\left(F_{1}, I \otimes_{R} Z\right)$.

Since $\varphi_{F_{0}}$ and $\varphi_{F_{1}}$ are clearly isomorphisms, so is $\varphi_{M}$.

The special case where $I=R$ is particularly interesting. We deduce

Lemma 3.2. Let $M$ be a finitely presented $R$-module, let $Z$ be a flat $R$-module, and let $f: M \longrightarrow Z$ be a homomorphism. Then $f$ factors as $M \longrightarrow$ $F \longrightarrow Z$, where $F$ is finitely generated and projective.

Proof. Consider the special case of Lemma 3.1, where $I=R$. Lemma 3.1 tells us that the map

$$
\varphi_{M}: \operatorname{Hom}_{R}(M, R) \otimes_{R} Z \longrightarrow \operatorname{Hom}_{R}(M, Z)
$$

is an isomorphism; in particular $\varphi_{M}$ is surjective. Any map $M \longrightarrow Z$ must lie in the image of $\varphi_{M}$, that is, any map can be expressed as the image of some finite sum $\sum_{i=1}^{n} f_{i} \otimes z_{i}$. But this means that the map factors as

$$
M \stackrel{\left(\begin{array}{c}
f_{1} \\
f_{2} \\
\vdots \\
f_{n}
\end{array}\right)}{\longrightarrow} R^{n} \stackrel{\left(\begin{array}{llll}
z_{1} & z_{2} & \cdots & z_{n}
\end{array}\right)}{\longrightarrow} Z .
$$

The form in which we will want to use this, again and again, is the following:

Corollary 3.3. Suppose $X$ and $Y$ are finitely generated projective modules, and $Z$ is a flat module. Suppose we are given homomorphisms

$$
X \stackrel{f}{\longrightarrow} Y \stackrel{g}{\longrightarrow} Z
$$

with $g f=0$. Then the map $g: Y \longrightarrow Z$ can be factored as $Y \stackrel{\rho}{\longrightarrow}$ $W \stackrel{\sigma}{\longrightarrow} Z$, that is we can extend the sequence of maps to

$$
X \stackrel{f}{\longrightarrow} Y \stackrel{\rho}{\longrightarrow} W \stackrel{\sigma}{\longrightarrow} Z,
$$

in such a way that: 
(i) The module $W$ is finitely generated and projective.

(ii) The composite

$$
X \stackrel{f}{\longrightarrow} Y \stackrel{\rho}{\longrightarrow} W
$$

vanishes.

Proof. Because the composite $g f: X \longrightarrow Z$ vanishes, the map $g$ : $Y \longrightarrow Z$ must factor through the cokernel $Q$ of $f$. That is, there is a short exact sequence

$$
X \stackrel{f}{\longrightarrow} Y \stackrel{\beta}{\longrightarrow} Q \longrightarrow 0,
$$

and a factorization of $g$ as

$$
Y \stackrel{\beta}{\longrightarrow} Q \stackrel{\tau}{\longrightarrow} Z
$$

Now $\tau: Q \longrightarrow Z$ is a map from the finitely presented module $Q$ to the flat module $Z$, and Lemma 3.2 tells us that it must factor as

$$
Q \stackrel{\gamma}{\longrightarrow} W \stackrel{\sigma}{\longrightarrow} Z,
$$

with $W$ finitely generated and projective. That is, the map $g: Y \longrightarrow Z$ has now been factored as

$$
Y \stackrel{\gamma \beta}{\longrightarrow} W \stackrel{\sigma}{\longrightarrow} Z
$$

we leave it to the reader to check that this factorization has the desired properties.

\section{An $\aleph_{1}$-perfect set of $\aleph_{1}$-small objects}

In this section we will study a certain set of objects in $\mathbf{K}(R$-Proj), which we will eventually prove to be an $\aleph_{1}$-compact generating set. Being objects in $\mathbf{K}(R$-Proj), they must be complexes of projective $R$-modules. The particular complexes we have in mind will be bounded below complexes of finitely generated, projective modules. We will describe them in Construction 4.3.

First it will be handy to prove a couple of little lemmas about chain maps from chain complexes of finitely generated, projective $R$-modules.

Lemma 4.1. Let $X$ be an object in $\mathbf{K}(R$-proj), and let $Z$ be an object of $\mathbf{K}(R$-Flat). Suppose we are given a chain map $f: X \longrightarrow Z$, that is a diagram

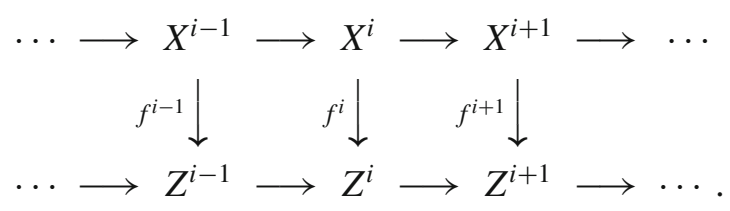

Suppose further that we are given some integer $j$, and that $f^{i}$ vanishes if $i<j$. Then the map $f$ factors, in the category $\mathbf{C}(R$-Mod), as $X \longrightarrow$ 
$Y \longrightarrow Z$, with $Y$ an object of $\mathbf{C}(R$-proj $)$, and $Y$ may be chosen so that $Y^{i}=0$ if $i<j$.

Proof. Without loss of generality we may assume $j=0$. If $i<0$ then the map $f^{i}: X^{i} \longrightarrow Z^{i}$ vanishes by hypothesis, and therefore factors through $Y^{i}=0$. It therefore suffices to show, by induction, that if we can define a factorization up to some integer $i$, then we can extend to $i+1$.

Consider therefore the commutative diagram, obtained because of the induction hypothesis

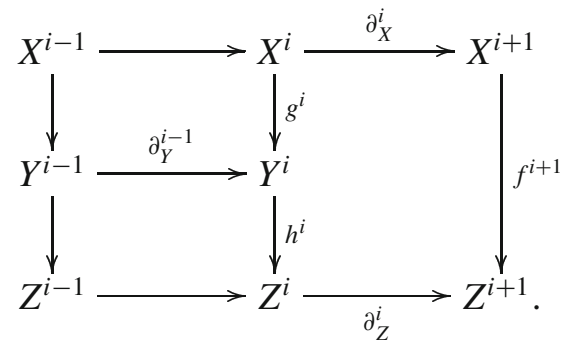

An easy diagram chase tells us that the maps

$$
Y^{i-1} \oplus X^{i} \stackrel{\left(\begin{array}{cc}
\partial_{Y}^{i-1} & -g^{i} \\
0 & \partial_{X}^{i}
\end{array}\right)}{\longrightarrow} Y^{i} \oplus X^{i+1} \stackrel{\left(\partial_{Z}^{i} h^{i}, f^{i+1}\right)}{\longrightarrow} Z^{i+1}
$$

must compose to zero. We are in the situation of Corollary 3.3; the modules $Y^{i-1} \oplus X^{i}$ and $Y^{i} \oplus X^{i+1}$ are finitely generated and projective, and the module $Z^{i+1}$ is flat. There is therefore a factorization of the map $\left(\partial_{Z}^{i} h^{i}, f^{i+1}\right)$ : $Y^{i} \oplus X^{i+1} \longrightarrow Z^{i+1}$ as

$$
Y^{i} \oplus X^{i+1} \stackrel{\left(\partial_{Y}^{i}, g^{i+1}\right)}{\longrightarrow} Y^{i+1} \stackrel{h^{i+1}}{\longrightarrow} Z^{i+1},
$$

where $Y^{i+1}$ is finitely generated and projective, and the composite

$$
Y^{i-1} \oplus X^{i} \stackrel{\left(\begin{array}{cc}
\partial_{Y}^{i-1} & -g^{i} \\
0 & \partial_{X}^{i}
\end{array}\right)}{\longrightarrow} Y^{i} \oplus X^{i+1} \stackrel{\left(\partial_{Y}^{i}, g^{i+1}\right)}{\longrightarrow} Y^{i+1}
$$

vanishes. This defines for us the maps $g^{i+1}, h^{i+1}$ and $\partial_{Y}^{i}$ in the diagram

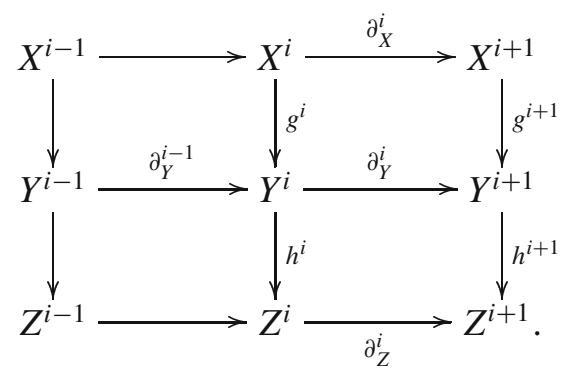


We leave it to the reader to check the commutativity of the diagram, to check that $h^{i+1} g^{i+1}=f^{i+1}$, and to verify that $\partial_{Y}^{i} \partial_{Y}^{i-1}=0$.

Lemma 4.2. Let $X$ and $Y$ be objects in $\mathbf{C}(R$-proj), let $Z$ be an object in $\mathbf{C}(R$-Flat), and let $X \longrightarrow Y \longrightarrow Z$ be two chain maps whose composite vanishes in $\mathbf{C}\left(R\right.$-Flat). Suppose also that $X^{i}=0$ for all $i \ll 0$. Then there exists an object $W$ of $\mathbf{C}(R$-proj), and a factorization, in $\mathbf{C}(R$-Flat $)$, of the chain map $Y \longrightarrow Z$ as $Y \longrightarrow W \longrightarrow Z$, so that the composite $X \longrightarrow Y \longrightarrow W$ vanishes. Furthermore, the maps $Y^{i} \longrightarrow W^{i}$ may be chosen to be isomorphisms if $i \ll 0$.

Proof. We know that $X^{i}=0$ for $i \ll 0$; without loss of generality we may suppose that $X^{i}=0$ for all $i \leq 0$. For $i \leq 0$ we define $W^{i}=Y^{i}$, and the map $Y \longrightarrow W$ is given, for $i \leq 0$, by the identity $1: Y^{i} \longrightarrow Y^{i}=W^{i}$.

Next we extend to all $i$. As in Lemma 4.1, we proceed by induction. We know that the composite $X \longrightarrow Y \longrightarrow Z$ vanishes. What we will do, by induction on $i$, is factorize $Y^{i} \longrightarrow Z^{i}$ as $Y^{i} \longrightarrow W^{i} \longrightarrow Z^{i}$, in such a way that the composite $X^{i} \longrightarrow Y^{i} \longrightarrow W^{i}$ vanishes. Note that for $i \leq 0$ we know that $X^{i}=0$, hence any composite $X^{i} \longrightarrow Y^{i} \longrightarrow W^{i}$ will vanish, and the choice of $W^{i}=Y^{i}$, made in the previous paragraph, works fine. There is no problem starting the induction.

We need to show how to extend from $i$ to $i+1$. Much as in the proof of Lemma 4.1, we consider the commutative diagram

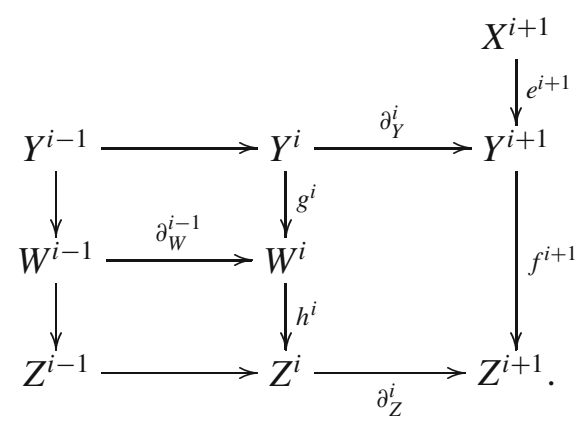

We know that $f^{i+1} e^{i+1}=0$; an easy diagram chase establishes the vanishing of the composite

$W^{i-1} \oplus Y^{i} \oplus X^{i+1} \stackrel{\left(\begin{array}{ccc}\partial_{W}^{i-1} & -g^{i} & 0 \\ 0 & \partial_{Y}^{i} & e^{i+1}\end{array}\right)}{\longrightarrow} W^{i} \oplus Y^{i+1} \stackrel{\left(\partial_{Z}^{i} h^{i}, f^{i+1}\right)}{\longrightarrow} Z^{i+1}$.

Corollary 3.3 applies; the modules $W^{i-1} \oplus Y^{i} \oplus X^{i+1}$ and $W^{i} \oplus Y^{i+1}$ are finitely generated and projective, while the module $Z^{i+1}$ is flat. We deduce that the map $\left(\partial_{Z}^{i} h^{i}, f^{i+1}\right): W^{i} \oplus Y^{i+1} \longrightarrow Z^{i+1}$ must factor through

$$
W^{i} \oplus Y^{i+1} \stackrel{\left(\partial_{W}^{i}, g^{i+1}\right)}{\longrightarrow} W^{i+1} \stackrel{h^{i+1}}{\longrightarrow} Z^{i+1},
$$


where $W^{i+1}$ is finitely generated and projective, and the composite

$$
W^{i-1} \oplus Y^{i} \oplus X^{i+1} \stackrel{\left(\begin{array}{ccc}
\partial_{W}^{i-1} & -g^{i} & 0 \\
0 & \partial_{Y}^{i} & e^{i+1}
\end{array}\right)}{\longrightarrow} W^{i} \oplus Y^{i+1} \stackrel{\left(\partial_{W}^{i}, g^{i+1}\right)}{\longrightarrow} W^{i+1}
$$

vanishes. This defines for us the maps $g^{i+1}, h^{i+1}$ and $\partial_{W}^{i}$ in the diagram

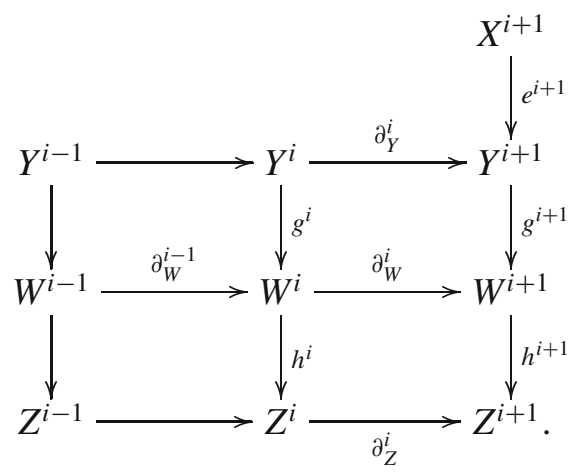

We leave it to the reader to check the commutativity of the diagram, to check that $h^{i+1} g^{i+1}=f^{i+1}$, and to verify that $g^{i+1} e^{i+1}=0$ and $\partial_{W}^{i} \partial_{W}^{i-1}=0$.

Construction 4.3. Let $R$ be a ring. Consider the category $\mathbf{C}^{+}(R$-proj); the objects are chain complexes $X$, that is complexes

$$
\cdots \longrightarrow X^{i-1} \longrightarrow X^{i} \longrightarrow X^{i+1} \longrightarrow \cdots
$$

where each $X^{i}$ is a finitely generated, projective $R$-module, and $X^{i}=0$ for all $i \ll 0$. The morphisms are the honest chain maps, not homotopy equivalence classes. Let $T$ be a set containing one representative in each $\mathbf{C}^{+}(R$-proj)-isomorphism class of objects.

Remark 4.4. Let us now pass from $\mathbf{C}^{+}(R$-proj) to the associated homotopy category $\mathbf{K}^{+}(R$-proj). In Construction 4.3 we defined $T$ to be a set containing (a hugh number of) representatives for each isomorphism class of objects in $\mathbf{K}^{+}\left(R\right.$-proj); we note that $\mathbf{K}^{+}(R$-proj) is a triangulated subcategory of $\mathbf{K}(R$-Flat $)$.

Lemma 4.5. Let $T$ be as in Construction 4.3. The objects in $T$ are all $\aleph_{1}$-small, in the category $\mathbf{K}(R$-Flat $)$.

Proof. Let $X$ be an object of $T$, and let $\left\{Z_{\lambda}, \lambda \in \Lambda\right\}$ be a set of objects in $\mathbf{K}(R$-Flat $)$. Suppose we are given a map

$$
X \rightarrow \coprod_{\lambda \in \Lambda} Z_{\lambda}
$$


We need to produce a factorization through

$$
X \longrightarrow \coprod_{\lambda \in \Lambda^{\prime}} Z_{\lambda} \stackrel{\text { canonical inclusion }}{\longrightarrow} \coprod_{\lambda \in \Lambda} Z_{\lambda},
$$

where the cardinality of $\Lambda^{\prime} \subset \Lambda$ is less than $\aleph_{1}$, that is where $\Lambda^{\prime}$ is countable.

Now our map of chain complexes comes down to

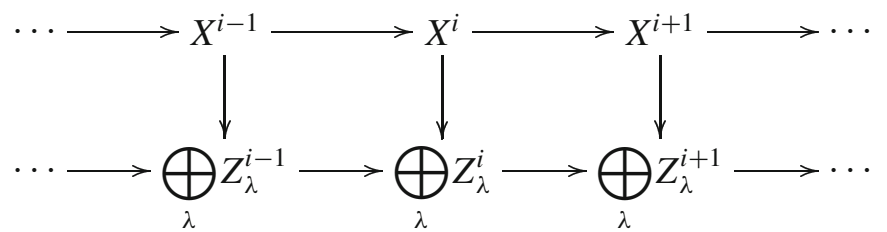

and the point is that, in each degree, we get a map $X^{i} \longrightarrow \bigoplus Z_{\lambda}^{i}$, where $X^{i}$ is finitely generated and projective. Let us fix the integer $i$ for a second. For our fixed $i$, it follows that only finitely many components can fail to vanish. For all but finitely many $\lambda \in \Lambda$, the component $X^{i} \longrightarrow Z_{\lambda}^{i}$ must vanish. There are countably many integers $i$, and for each $i$ we have finitely many possibilities of $\lambda$ where $X^{i} \longrightarrow Z_{\lambda}^{i}$ might be non-zero. Hence the factorization through a countable direct sum.

Lemma 4.6. Let $T$ be as in Construction 4.3. Then the set $T$ is $\aleph_{1}$-perfect, in the category $\mathbf{K}$ ( $R$-Flat).

Remark 4.7. Suppose $X$ is an object of $T$. To say that $T$ is $\aleph_{1}$-perfect implies, among other things, that any map from $X$ to a countable coproduct of objects in $\mathbf{K}(R$-Flat), that is any map

$$
X \longrightarrow \coprod_{\lambda=1}^{\infty} Z_{\lambda}
$$

must factor as

$$
X \longrightarrow \coprod_{\lambda=1}^{\infty} Y_{\lambda} \stackrel{\bigsqcup_{\lambda=1}^{\infty} f_{\lambda}}{\longrightarrow} \coprod_{\lambda=1}^{\infty} Z_{\lambda}
$$

with $Y_{\lambda} \in T$. We will prove more. The refinement we will show, in the course of the proof of Lemma 4.6, says

Refinement 4.8. In the factorization

$$
X \longrightarrow \coprod_{\lambda=1}^{\infty} Y_{\lambda} \stackrel{\bigsqcup_{\lambda=1}^{\infty} f_{\lambda}}{\longrightarrow} \coprod_{\lambda=1}^{\infty} Z_{\lambda}
$$

of Remark 4.7, the objects $Y_{\lambda} \in T$ may be chosen so that, for any integer $j$, there are only finitely many pairs of integers $(i, \lambda)$, with $i<j$ and $1 \leq$ $\lambda<\infty$, for which $Y_{\lambda}^{i} \neq 0$. 
Proof. We begin with a slight refinement of the argument in Lemma 4.5. Any morphism, from an object $X$ in $T$ to a (countable) sum of objects in $\mathbf{K}(R$-Flat $)$, must come from a chain map

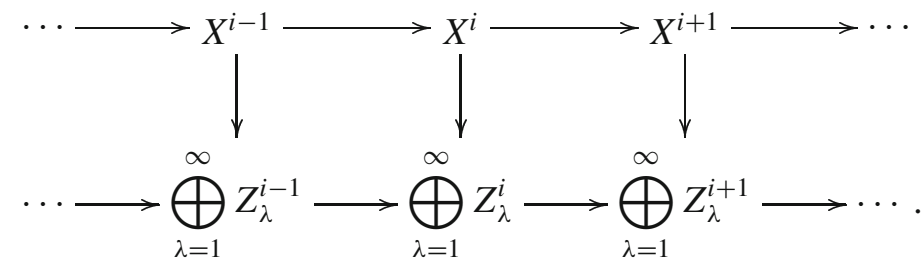

Let us fix this chain map. The chain map has the property that, in each degree, all but finitely many of the components vanish. That is the map $X^{i} \longrightarrow \bigoplus Z_{\lambda}^{i}$ factors through a finite sum. We also know, from the fact that $X$ is in $T$ and from Construction 4.3, that $X^{i}=0$ for all $i \ll 0$. Hence for each $j \in \mathbb{Z}$ there exist finitely many $i<j$ with $X^{i} \neq 0$, and for each of those there are finitely many $\lambda \in \mathbb{N}$ with $X^{i} \longrightarrow Z_{\lambda}^{i}$ non-zero. That is, for all but finitely many $\lambda \in \mathbb{N}$, the chain map $X \longrightarrow Z_{\lambda}$ is zero in degrees $<j$.

For each integer $j \in \mathbb{Z}$, let $\Lambda_{j} \subset \mathbb{N}$ be the finite set of all $\lambda \in \mathbb{N}$, so that $X^{i} \longrightarrow Z_{\lambda}^{i}$ is non-zero for some $i<j$. In symbols

$$
\Lambda_{j}=\left\{\lambda \in \mathbb{N} \mid \exists i<j \text { with } X^{i} \longrightarrow Z_{\lambda}^{i} \text { non-zero }\right\} .
$$

The sets $\Lambda_{j}$ clearly increase with $j$; that is, $\Lambda_{j} \subset \Lambda_{j+1}$. Now take any $\lambda \in \Lambda_{j+1}-\Lambda_{j}$; because $\lambda \notin \Lambda_{j}$, the chain map $X \longrightarrow Z_{\lambda}$ must vanish in degrees $<j$. By Lemma 4.1 we deduce that it has a factorization $X \longrightarrow Y_{\lambda} \longrightarrow Z_{\lambda}$, with $Y_{\lambda}$ a complex of finitely generated projective modules, vanishing in degrees $<j$. In particular, $Y_{\lambda}$ is isomorphic to an object in $T$; replacing $Y_{\lambda}$ by an isomorph, we may assume $Y_{\lambda} \in T$. This defines $Y_{\lambda}$, whenever $\lambda \in \bigcup_{j \in \mathbb{Z}} \Lambda_{j}$; for $\lambda$ outside the union $\bigcup_{j \in \mathbb{Z}} \Lambda_{j}$, we define $Y_{\lambda}=0$.

But now the map $X \longrightarrow \coprod_{\lambda=1}^{\infty} Z_{\lambda}$ factors, in the category $\mathbf{C}(R$-Mod), as

$$
X \longrightarrow \coprod_{\lambda=1}^{\infty} Y_{\lambda} \longrightarrow \coprod_{\lambda=1}^{\infty} Z_{\lambda},
$$

where the reader is asked to observe that, in each degree $j \in \mathbb{Z}$, the direct sum $\bigsqcup_{\lambda=1}^{\infty} Y_{\lambda}^{j}$ is actually finite, and hence the map from $X^{j}$ into it is welldefined. This proves Refinement 4.8. It remains to show that Lemma 4.6 follows from Refinement 4.8 .

In Remark 4.4 we noted that the set $T$ consists of (many) representatives for the isomorphism classes of objects in a triangulated subcategory $\mathbf{K}^{+}(R$-Proj $) \subset \mathbf{K}(R$-Flat $)$. We have just proved that every object in $T$ is $\aleph_{1}$-good, as in [11, Definition 3.3.4]. From [11, Lemma 3.3.5 and Remark 3.3.6] we conclude that the set $T$ is $\aleph_{1}$-perfect. 


\section{Generators for the category $\mathbf{K}(R$-Proj)}

In Sect. 4 we constructed a set $T$ of objects in $\mathbf{K}(R$-proj), and showed that $T$ is an $\aleph_{1}$-perfect set of $\aleph_{1}$-small objects in $\mathbf{K}(R$-Flat $)$. Since the objects of $T$ all lie in the subcategory $\mathbf{K}(R$-Proj $) \subset \mathbf{K}(R$-Flat $)$, and the subcategory $\mathbf{K}(R$-Proj) is triangulated and closed under the formation in $\mathbf{K}(R$-Flat) of coproducts of its objects, the set $T$ can only generate the subcategory. The main result of this section is that it does.

Remark 5.1. Unfortunately the proof we will give is by first choosing bases. It would be nice to have a basis-free, conceptual argument; it goes without saying that, if I had such a proof, I would gladly toss out the one presented in this section and replace it by the more elegant approach.

We will prove that $T$ generates $\mathbf{K}(R$-Proj) by means of a sequence of lemmas, some of which will be needed later. First we remind the reader of the notion of the rank of a projective module.

Reminder 5.2. Let $P$ be a projective $R$-module. Then there exists an $R$-module $Q$, and an isomorphism $P \oplus Q \simeq R^{\beta}$, where $R^{\beta}$ is a direct sum of $\beta$ copies of $R$. If, for a given cardinal $\beta$, there exists a $Q$ and an isomorphism $P \oplus Q \simeq R^{\beta}$, then we say that $P$ is a projective $R$-module of rank $\leq \beta$. The rank of $P$ is the smallest $\beta$ that works; it exists because the cardinals are well-ordered.

Given any set of projective $R$-modules, there exists a cardinal $\beta$ larger than all their ranks; just take the sum of the ranks. In particular, given a chain complex $Z$ of projective $R$-modules, there exists a cardinal $\beta$ so that, for each $i \in \mathbb{Z}$, the rank of $Z^{i}$ is $\leq \beta$.

Lemma 5.3. Let $Z$ be an object of $\mathbf{K}(R$-Proj), that is $Z$ is a complex of projective modules. Assume that $\alpha$ is some infinite cardinal, and all the projective modules $Z^{i}$ are of rank $\leq \alpha$. Then $Z$ is homotopy equivalent to $\bar{Z}$, where $\bar{Z}$ is a chain complex of free modules of rank $\leq \alpha$.

Proof. We are given a complex $Z$ of projective modules

$$
\cdots \longrightarrow Z^{i-1} \longrightarrow Z^{i} \longrightarrow Z^{i+1} \longrightarrow \cdots
$$

and we are told that each $Z^{i}$ is of rank $\leq \alpha$. This means that, for each $i \in \mathbb{Z}$, we can choose a module $Y^{i}$ so that $Z^{i} \oplus Y^{i}$ is free, and of rank $\leq \alpha$. Put

$$
F(i)=\bigoplus_{1}^{\infty}\left(Z^{i} \oplus Y^{i}\right)
$$

Then $F(i)$ is a countable direct sum of free modules of rank $\leq \alpha$, and hence it is free of rank $\leq \alpha$. Also,

$$
Z^{i} \oplus F(i)=Z^{i} \oplus\left(\bigoplus_{1}^{\infty} Z^{i}\right) \oplus\left(\bigoplus_{1}^{\infty} Y^{i}\right) \cong\left(\bigoplus_{1}^{\infty} Z^{i}\right) \oplus\left(\bigoplus_{1}^{\infty} Y^{i}\right)=F(i),
$$


and hence $Z^{i} \oplus F(i)$ is also free, of rank $\leq \alpha$. Now let $C(i)$ be the chain complex

$$
\cdots \longrightarrow 0 \longrightarrow F(i)^{i-1} \stackrel{1}{\longrightarrow} F(i)^{i} \longrightarrow 0 \longrightarrow \cdots .
$$

This notation means that $C(i)$ is the chain complex

$$
\cdots \longrightarrow 0 \longrightarrow F(i) \stackrel{1}{\longrightarrow} F(i) \longrightarrow 0 \longrightarrow \cdots
$$

where the $F(i)$ are placed in degrees $i-1$ and $i$. Now form the complex

$$
\bar{Z}=Z \oplus \bigoplus_{i=-\infty}^{\infty} C(i) .
$$

It is a direct sum of $Z$ with a contractible complex, hence is homotopy equivalent to $Z$. On the other hand, the $i^{\text {th }}$ term in this chain complex is $Z^{i} \oplus F(i) \oplus F(i+1)$. Both $Z^{i} \oplus F(i)$ and $F(i+1)$ are free of rank $\leq \alpha$, and the lemma follows.

The advantage, of working with complexes of free modules, is that one can choose a basis. Let us formalize this as a definition.

Definition 5.4. An augmented complex of free modules is a chain complex of free modules

$$
\cdots \longrightarrow Z^{i-1} \longrightarrow Z^{i} \longrightarrow Z^{i+1} \longrightarrow \cdots
$$

and, for every $i$, a choice of elements $e_{\lambda}^{i} \in Z^{i}$ which freely generate the module. An augmented subcomplex of the augmented complex $Z$ is a subcomplex $Y \subset Z$, where each $Y^{i} \subset Z^{i}$ is free, and the basis which augments $Y^{i}$ is a subset of the basis given for $Z^{i}$.

The next two lemmas allow us to construct augmented subcomplexes.

Lemma 5.5. Let $Z$ be an augmented complex of free modules. Suppose that, for each $i \in \mathbb{Z}$, we are given some finite subset $S_{i}$ of the basis giving the augmentation of $Z^{i}$. Suppose further that $S_{i}=\emptyset$ if $i \ll 0$.

Then there exists an augmented subcomplex $Y \subset Z$, so that

(i) Each $Y^{i}$ is of finite rank.

(ii) $S_{i} \subset Y^{i}$, for all $i \in \mathbb{Z}$.

(iii) $Y^{i}=0$ for all $i \ll 0$.

Proof. Let $j$ be the smallest integer with $S_{j} \neq \emptyset$. We will define, inductively on $i$, a finite subset $T_{i}$ of the basis giving the augmentation for $Z^{i}$. The definition is as follows:

(i) If $i<j$, put $T_{i}=\emptyset$.

(ii) Given $T_{i}$, we want to define $T_{i+1}$. We have a linear map $\partial^{i}: Z^{i} \longrightarrow Z^{i+1}$. For each $t \in T_{i} \subset Z^{i}$, its image $\partial^{i}\left(t_{i}\right)$ can be expanded as a linear combination in the chosen basis elements of $Z^{i+1}$. Finitely many basis elements occur. Let $T_{i+1}$ be the union of $S_{i+1}$ with the finitely many basis elements in the expansions of $\left\{\partial^{i}(t), t \in T_{i}\right\}$. 
Now let $Y^{i}$ be the free submodule of $Z^{i}$ with basis $T_{i}$; we leave it to the reader to check the assertions of the lemma.

Lemma 5.6. Let $Z$ be an augmented complex of free modules. Assume that we are given an augmented subcomplex $Y \subset Z$, that for each $i \in \mathbb{Z}$ we are given a set $S_{i}$ of elements of the preferred basis for $Z^{i}$, and further that we are given a homotopy $\Phi: Y \Longrightarrow Z$. Suppose that we make the following finiteness assumptions:

(i) Each $Y^{i}$ is of finite rank.

(ii) $Y^{i}=0$ for $i \ll 0$.

(iii) Each $S_{i}$ is finite.

(iv) $S_{i}=\emptyset$ for $i \ll 0$.

Then there exists an augmented subcomplex $\bar{Y} \subset Z$, containing $Y$, so that

(v) $\Phi: Y \Longrightarrow Z$ factors as $Y \Longrightarrow \bar{Y} \subset Z$.

(vi) $S_{i}$ is contained in $\bar{Y}$.

(vii) Each $\bar{Y}^{i}$ is of finite rank.

(viii) $\bar{Y}^{i}=0$ for all $i \ll 0$.

Proof. Let $A_{i}$ be the augmented basis for $Y^{i}$; because $Y^{i}$ is of finite rank, $A_{i}$ must be finite. The homotopy $\Phi^{i}: Y^{i} \longrightarrow Z^{i-1}$ takes any element $a \in A_{i}$ to $\Phi^{i}(a) \in Z^{i-1}$, which can be expressed as a finite linear combination of the basis elements of $Z^{i-1}$. Let $\bar{S}_{i}$ be the union of $A_{i} \cup S_{i}$ with the finite number of basis elements that occur in the expansion of $\left\{\Phi^{i+1}(a), a \in A_{i+1}\right\}$. Clearly the sets $\bar{S}_{i}$ are all finite, and are empty if $i \ll 0$.

Lemma 5.5 applies, for the finite sets $\bar{S}_{i} \subset Z^{i}$. It gives us an augmented subcomplex $\bar{Y} \subset Z$. We have, from Lemma 5.5, that $\bar{Y}^{i}$ are all of finite rank, that $\bar{Y}^{i}=0$ if $i \ll 0$, and that $\bar{S}_{i} \subset \bar{Y}^{i}$. Since $A_{i}$ is a basis for $Y^{i}$, and $A_{i} \subset \bar{S}_{i} \subset \bar{Y}^{i}$, it follows that $Y^{i} \subset \bar{Y}^{i}$. Also $S_{i}$ is contained in $\bar{S}_{i} \subset Y^{i}$. Furthermore, by construction of $\bar{S}_{i}$, the map $\Phi^{i+1}: Y^{i+1} \longrightarrow Z^{i}$ takes the basis $A_{i+1}$ of $Y^{i+1}$ into $\bar{S}_{i} \subset \bar{Y}^{i}$; therefore the homotopy $\Phi: Y \Longrightarrow Z$ has its image inside $\bar{Y} \subset Z$.

Lemma 5.7. Let $Z$ be an augmented complex of free modules and, for each integer $i \in \mathbb{Z}$, let $Q_{i} \subset Z^{i}$ be a subset of the basis that comes with the augmentation. Assume that each $Q_{i}$ is finite or countable. Then there exists a sequence of augmented subcomplexes

$$
Y_{0} \subset Y_{1} \subset Y_{2} \subset Y_{3} \subset \cdots
$$

so that

(i) Each $Y_{n}^{i}$ is of finite rank.

(ii) For each $n, Y_{n}^{i}=0$ if $i \ll 0$.

(iii) Put $Y=\bigcup_{n=0}^{\infty} Y_{n}$. Then $Q_{i}$ is contained in $Y^{i}$, for each $i \in \mathbb{Z}$.

If we further assume that, for any object $X \in T$, any map $X \longrightarrow Z$ is null homotopic, then the complex $Y$ can be chosen to be contractible. 
Proof. For each $i \in \mathbb{Z}$ we are given a subset $Q_{i} \subset Z^{i}$, which is no larger than countable. It follows that the set $\bigsqcup_{i=-\infty}^{\infty} Q_{i}$ is countable (or finite). Enumerate the elements; that is,

$$
\bigsqcup_{i=-\infty}^{\infty} Q_{i}=\left\{q_{0}, q_{1}, q_{2}, \ldots\right\} .
$$

By Lemma 5.5 there exists an augmented subcomplex $Y_{0}$ containing $q_{0}$. Furthermore, $Y_{0}$ satisfies the finiteness hypotheses of (i) and (ii). Next we apply induction and Lemma 5.6. Suppose we have an augmented subcomplex $Y_{n} \subset Z$, satisfying the finiteness hypotheses of (i) and (ii). Define $S_{i} \subset Z^{i}$ to be the finite set of basis vectors

$$
S_{i}= \begin{cases}\left\{q_{n+1}\right\} & \text { if } q_{n+1} \in Z^{i} \\ \varnothing & \text { otherwise }\end{cases}
$$

Let $\Phi: Y_{n} \Longrightarrow Z$ be the zero homotopy. Lemma 5.6 applies to the subcomplex $Y_{n}$, the sets $S_{i}$ and the zero homotopy, and gives us an augmented subcomplex $Y_{n+1}$. The subcomplex $Y_{n+1}$ satisfies the finiteness hypotheses (i) and (ii), and contains both $Y_{n}$ and $q_{n+1}$. It follows that $Y=\bigcup_{n=0}^{\infty} Y_{n}$ contains all the $q_{n}$; that is, $Q_{i} \subset Y^{i}$. It remains only to prove the "furthermore" statement.

Assume therefore that, for all objects $X$ in $T$, any map $X \longrightarrow Z$ is null homotopic. As before, Lemma 5.5 gives us an augmented subcomplex $Y_{0}$ containing $q_{0}$. The complex $Y_{0}$ satisfies the finiteness hypotheses (i) and (ii), hence it is isomorphic to an object in $T$. Therefore the inclusion $Y_{0} \longrightarrow Z$ is null homotopic. Choose a homotopy $\Phi_{0}$ contracting the inclusion. We will now inductively construct augmented subcomplexes $Y_{n} \subset Z$, and homotopies $\Phi_{n}: Y_{n} \Longrightarrow Z$ contracting the inclusions. The construction will be such that

(iv) The subcomplex $Y_{n+1}$ contains $Y_{n}$ and $q_{n+1}$.

(v) The homotopy $\Phi_{n}: Y_{n} \Longrightarrow Z$ factors as $Y_{n} \Longrightarrow Y_{n+1} \subset Z$.

(vi) Let $i$ be the inclusion $i: Y_{n} \longrightarrow Y_{n+1}$. The composite

$$
Y_{n} \stackrel{i}{\longrightarrow} Y_{n+1} \stackrel{\Phi_{n+1}}{\longrightarrow} Z
$$

agrees with $\Phi_{n}: Y_{n} \Longrightarrow Z$.

We have $Y_{0}$ and a homotopy $\Phi_{0}: Y_{0} \Longrightarrow Z$. We need to prove the induction step. Suppose therefore that we are given the augmented subcomplex $Y_{n} \subset Z$, as well as the homotopy $\Phi_{n}: Y_{n} \Longrightarrow Z$ contracting the inclusion. As above, we define

$$
S_{i}= \begin{cases}\left\{q_{n+1}\right\} & \text { if } q_{n+1} \in Z^{i} \\ \varnothing & \text { otherwise. }\end{cases}
$$

Lemma 5.6 applies to the augmented subcomplex $Y_{n} \subset Z$, the sets $S_{i} \subset Z^{i}$ and the homotopy $\Phi_{n}: Y_{n} \Longrightarrow Z$, and gives us an augmented subcom- 
plex $Y_{n+1}$. By the assertions of Lemma 5.6, the finiteness conditions (i) and (ii) are satisfied by $Y_{n+1}$. Furthermore, $Y_{n+1}$ contains $Y_{n}$ and the sets $S_{i}$. Since $q_{n+1} \in S_{i}$ for some $i$, we have that $Y_{n+1}$ contains $q_{n+1}$. It follows that (iv) is true. And also part (v) comes immediately; the homotopy $\Phi_{n}: Y_{n} \Longrightarrow Z$ factors as $Y_{n} \Longrightarrow Y_{n+1} \subset Z$. It remains to define the homotopy $\Phi_{n+1}: Y_{n+1} \Longrightarrow Z$, and to prove (vi).

Since $Y_{n+1}$ satisfies the finiteness conditions (i) and (ii), it is isomorphic to an object in $T$. Hence any map $Y_{n+1} \longrightarrow Z$ is null homotopic; we may therefore choose a homotopy $\Psi: Y_{n+1} \Longrightarrow Z$, connecting the inclusion to the null map. However, if we make any dumb choice, there is no guarantee that the composite $Y_{n} \stackrel{i}{\longrightarrow} Y_{n+1} \stackrel{\Psi}{\Longrightarrow} Z$ will agree with $\Phi_{n}: Y_{n} \Longrightarrow Z$. Next we change $\Psi$ to achieve the good result.

Now $\Phi_{n}$ and $\Psi i$ are two homotopies $\Phi_{n}, \Psi i: Y_{n} \Longrightarrow Z$, contracting the same map. Hence $\Psi i-\Phi_{n}$ is a chain map $\Sigma Y_{n} \longrightarrow Z$. We know that $\Sigma Y_{n}$ is isomorphic to an object in $T$, and hence that any chain map $\Sigma Y_{n} \longrightarrow Z$ is null homotopic. Choose a homotopy $\Theta: \Sigma Y_{n} \Longrightarrow Z$, contracting the chain map $\Psi i-\Phi_{n}$. Because each $Y_{n}^{i}$ is a direct summand of $Y_{n+1}^{i}$, we can extend $\Theta$ to a homotopy $\bar{\Theta}: \Sigma Y_{n+1} \Longrightarrow Z$. Now $\rho=\bar{\Theta} \partial+\partial \bar{\Theta}$ is a chain map $\rho: \Sigma Y_{n+1} \longrightarrow Z$, and the composite

$$
\Sigma Y_{n} \stackrel{\Sigma i}{\longrightarrow} \Sigma Y_{n+1} \stackrel{\rho=\bar{\Theta} \partial+\partial \bar{\Theta}}{\longrightarrow} Z
$$

agrees with $\Psi i-\Phi_{n}$. In other words, $\Phi_{n+1}=\Psi-\rho$ is a homotopy $Y_{n+1} \Longrightarrow Z$, with $\Phi_{n+1} i=(\Psi-\rho) i=\Phi_{n}$. Further, because $\rho$ : $\Sigma Y_{n+1} \longrightarrow Z$ is a chain map, $\partial \rho+\rho \partial=0$; this makes

$$
\partial \Phi_{n+1}+\Phi_{n+1} \partial=\partial \Psi+\Psi \partial,
$$

which is the inclusion $Y_{n+1} \longrightarrow Z$.

The contracting homotopies $\Phi_{n}: Y_{n} \Longrightarrow Y_{n+1}$ are compatible, and hence assemble together to a homotopy $\Phi: Y \Longrightarrow Y$, where $Y=\bigcup Y_{n}$ is the union. We deduce that $Y$ is contractible.

Corollary 5.8. Suppose $Z$ is an augmented complex of free modules and $Z$ is non-zero in $\mathbf{C}(R$-Mod). Suppose further that, for any object $X \in T$, any map $X \longrightarrow Z$ is null homotopic. Then there is a non-zero augmented subcomplex $Y \subset Z$, and $Y$ is contractible.

Proof. Because $Z \neq 0$, there is some $k \in \mathbb{Z}$ for which $Z^{k} \neq 0$, and hence the basis for $Z^{k}$ cannot be empty. For every $j \in \mathbb{Z}$ choose a finite subset $Q_{j}$ of the basis of $Z^{j}$ by the rule

$$
Q_{j}= \begin{cases}\emptyset & \text { if } j \neq k \\ \text { nonempty } & \text { if } j=k .\end{cases}
$$

Lemma 5.7 applies, to give us an augmented subcomplex $Y \subset Z$, which is contractible and contains $Q$. Because $Q_{k} \subset Q$ is non-empty, $Y$ cannot vanish. 
Now we deduce the main theorem:

Theorem 5.9. Let $T$ be the set of objects of $\mathbf{K}(R$-proj), defined in Construction 4.3. The set $T$ forms an $\aleph_{1}$-compact set of generators for the category $\mathbf{K}(R$-Proj).

Proof. We already know, from Lemmas 4.5 and 4.6, that $T$ is an $\aleph_{1}$-perfect set of $\aleph_{1}$-small objects. It remains only to establish the generation. What we need to prove is the following: suppose $Z$ is an object of $\mathbf{K}(R$-Proj), and suppose that, for every object $X \in T$, every map $X \longrightarrow Z$ is null homotopic. Then $Z$ is contractible.

By Lemma 5.3, $Z$ is homotopy equivalent to a complex of free modules. Replacing $Z$ by a homotopy equivalent complex, we may assume that $Z$ is a complex of free modules, and we may choose an augmentation, meaning a basis for each $Z^{i}$. Let us choose and fix the basis.

Consider the set of all pairs $(Y, \Phi)$, where $Y$ is an augmented subcomplex of $Z$, and $\Phi$ is a contracting homotopy on $Y$. We make the collection of all pairs $(Y, \Phi)$ into an ordered set, by putting $(Y, \Phi) \leq\left(Y^{\prime}, \Phi^{\prime}\right)$ if $Y$ is an augmented subcomplex of $Y^{\prime}$, and the following square commutes

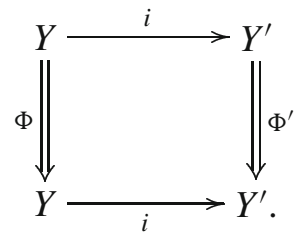

Here $i: Y \longrightarrow Y^{\prime}$ is the inclusion. In other words, the homotopy $\Phi^{\prime}$ : $Y^{\prime} \Longrightarrow Y^{\prime}$ restricts to the homotopy $\Phi: Y \Longrightarrow Y$. Now any totally ordered set of pairs $(Y, \Phi)$ is bounded above, by the union. Zorn's Lemma tells us that there is a maximal ordered pair $(Y, \Phi)$. It clearly suffices to prove that $Y=Z$.

In any case, we have a short exact sequence of augmented chain complexes

$$
0 \longrightarrow Y \longrightarrow Z \longrightarrow Z^{\prime} \longrightarrow 0
$$

this sequence is split in each degree. This means that $Z$ is the mapping cone on some map of chain complexes $\Sigma^{-1} Z^{\prime} \longrightarrow Y$. Since $Y$ is contractible, $Z$ and $Z^{\prime}$ are homotopy equivalent. It follows that, for any object $X \in T$, any map $X \longrightarrow Z^{\prime}$ is null homotopic.

Assume $Z^{\prime} \neq 0$, and we will prove a contradiction. Corollary 5.8 says that there is a non-zero augmented subcomplex $Y^{\prime} \subset Z^{\prime}$, which is contractible. The inverse image of $Y^{\prime} \subset Z^{\prime}$, under the surjection $Z \longrightarrow Z^{\prime}$, is the mapping cone on some map of chain complexes $\Sigma^{-1} Y^{\prime} \longrightarrow Y$. Since $Y$ and $Y^{\prime}$ are both contractible, there is a contraction of the mapping cone extending the contraction of $Y$. This contradicts the maximality of $(Y, \Phi)$. 
Corollary 5.10. The category $\mathbf{K}(R$-Proj) satisfies Brown representability. Furthermore, any localizing subcategory containing $T$ must be all of $\mathbf{K}(R$-Proj). In the notation of [11], $\langle T\rangle=\mathbf{K}(R$-Proj).

Proof. Theorem 5.9 tells us that $T$ is an $\aleph_{1}$-perfect generating set; the corollary follows from [11, Theorem 8.3.3]. Note that, for this corollary, we did not need the $\aleph_{1}$-smallness of the objects in $T$.

\section{Homotopy colimits in $\mathbf{K}(R-\mathrm{Mod})$}

In any triangulated category $\mathcal{T}$ with coproducts one has the notion of homotopy colimits. We remind the reader: given a sequence of objects and morphisms

$$
Y_{0} \longrightarrow Y_{1} \longrightarrow Y_{2} \longrightarrow Y_{3} \longrightarrow \cdots
$$

the homotopy colimit Hocolim $Y_{n}$ is defined, up to isomorphism, by the distinguished triangle

$$
\coprod_{n=0}^{\infty} Y_{n} \stackrel{1-\text { shift }}{\longrightarrow} \coprod_{n=0}^{\infty} Y_{n} \longrightarrow \underline{\text { Hocolim }} Y_{n} \longrightarrow \Sigma \coprod_{n=0}^{\infty} Y_{n} .
$$

What is most helpful are the situations in which we can identify a homotopy colimit with an ordinary colimit. In the category $\mathbf{K}(R$-Mod $)$, the useful criterion is the following.

Lemma 6.1. Suppose we are given a sequence of chain maps of chain complexes

$$
Y_{0} \longrightarrow Y_{1} \longrightarrow Y_{2} \longrightarrow Y_{3} \longrightarrow \cdots \text {. }
$$

Let $Y$ be the ordinary colimit. There is always a short exact sequence of chain complexes of $R$-modules

$$
0 \longrightarrow \coprod_{n=0}^{\infty} Y_{n} \stackrel{1-\text { shift }}{\longrightarrow} \coprod_{n=0}^{\infty} Y_{n} \longrightarrow Y \longrightarrow 0 .
$$

If, in each degree $i \in \mathbb{Z}$, the short exact sequence of $R$-modules splits, then $Y$ is the homotopy colimit of the sequence.

Remark 6.2. Note that Hocolim $Y_{n}$ is only defined up to non-canonical isomorphism; when we say that $Y$ "is" the homotopy colimit, the statement only makes sense up to isomorphism in $\mathbf{K}(R$-Mod), that is up to homotopy equivalence of chain complexes.

Proof. We know that, in each degree, the short exact sequence of chain complexes splits. This tells us that the middle term is the mapping cone on a chain map $\Sigma^{-1} Y \longrightarrow \bigsqcup_{n=0}^{\infty} Y_{n}$, and hence that $Y$ is homotopy equivalent to the mapping cone on the map 1-shift. 
Remark 6.3. Suppose we are given a sequence of chain maps of chain complexes

$$
Y_{0} \longrightarrow Y_{1} \longrightarrow Y_{2} \longrightarrow Y_{3} \longrightarrow \cdots .
$$

For each integer $i$, in degree $i$ we have a sequence of $R$-modules

$$
Y_{0}^{i} \longrightarrow Y_{1}^{i} \longrightarrow Y_{2}^{i} \longrightarrow Y_{3}^{i} \longrightarrow \cdots .
$$

Let $Y^{i}$ be the ordinary colimit. We are interested in conditions that guarantee that the short exact sequence of $R$-modules

$$
0 \longrightarrow \coprod_{n=0}^{\infty} Y_{n}^{i} \stackrel{1-\text { shift }}{\longrightarrow} \coprod_{n=0}^{\infty} Y_{n}^{i} \longrightarrow Y^{i} \longrightarrow 0
$$

splits. A sufficient criterion is that the $Y_{n}^{i}$ stabilize; that is there should exist an integer $j$, so that for all $n>j$ the map $Y_{n}^{i} \longrightarrow Y^{i}$ is an isomorphism.

A slightly fancier version is when the sequence $\left\{Y_{n}^{i}\right\}$ is a coproduct of sequences which are eventually stable. We will use the fancy version in the proof of Lemma 7.3, and the simple-minded variant in the proof of Proposition 7.14.

\section{The compact objects of $\mathbf{K}(R$-Proj)}

We now know that the category $\mathbf{K}(R$-Proj $)$ is an $\aleph_{1}$-compactly generated triangulated category. It becomes interesting to study what are the subcategories of compact objects. In this section we will compute the subcategories $\mathbf{K}(R \text {-Proj })^{\alpha}$ for every regular cardinal $\alpha$; note that $\mathbf{K}(R \text {-Proj })^{\aleph_{0}}$ is the subcategory that is usually referred to as $\mathbf{K}(R \text {-Proj })^{c}$, the (ordinary) compact objects. We will also study when the compact objects generate; it turns out that $\mathbf{K}(R$-Proj $)$ need not be compactly generated. It depends on $R$; for some $R$ it is compactly generated, for other $R$ it is not.

Lemma 7.1. The category $\mathbf{K}(R-\operatorname{Proj})^{\aleph_{1}}$ contains the set $T$ of Construction 4.3.

Proof. The set $T$ is an $\aleph_{1}$-perfect set of $\aleph_{1}$-small objects in $\mathbf{K}(R$-Proj $)$, and the category $\mathbf{K}(R \text {-Proj })^{\aleph_{1}}$ is the unique maximal such; it contains all others.

Lemma 7.2. Every object in $\mathbf{K}(R \text {-Proj })^{\aleph_{1}}$ is isomorphic, in $\mathbf{K}(R$-Proj), to a complex of free modules of rank $\leq \aleph_{0}$.

Proof. Let $\&$ be the full subcategory of $\mathbf{K}(R$-Proj), whose objects are all the isomorphs, in $\mathbf{K}(R$-Proj $)$, of complexes of free modules of rank $\leq \aleph_{0}$. The lemma comes down to saying that $\mathbf{K}(R \text {-Proj })^{\aleph_{1}} \subset \&$. Clearly $\&$ is 
triangulated, and closed in $\mathbf{K}(R$-Proj) under the formation of countable coproducts. It is an $\aleph_{1}$-localizing subcategory.

Next observe that $\delta$ contains $T$. Every object $X \in T$ is a chain complex of finitely generated, projective modules. Applying Lemma 5.3, with $\alpha=\aleph_{0}$, we conclude that $X$ is homotopy equivalent to an $\bar{X}$, where $\bar{X}$ is a complex of free modules of rank $\leq \aleph_{0}$. That is, $X$ is an object of $\&$.

By Lemma 7.1, $T$ is contained in $\mathbf{K}(R-\text { Proj })^{\aleph_{1}}$. Corollary 5.10 told us that $\langle T\rangle=\mathbf{K}$ (R-Proj). From [11, Lemma 4.4.5] it follows that $\langle T\rangle^{\aleph_{1}}=$ $\mathbf{K}(R \text {-Proj })^{\aleph_{1}}$. This notation means that any $\aleph_{1}$-localizing subcategory, which contains $T$, must contain all of $\mathbf{K}(R \text {-Proj })^{\aleph_{1}}$. Since $\delta$ is $\aleph_{1}$-localizing and contains $T$, we deduce the Lemma.

Next we want to prove the reverse inclusion; we wish to show that any chain complex, of free modules of rank $\leq \aleph_{0}$, lies in $\mathbf{K}(R \text {-Proj })^{\aleph_{1}}$. We prove the following, slightly stronger statement.

Lemma 7.3. Let $Y$ be a chain complex, of free modules of rank $\leq \aleph_{0}$. Then $Y$ can be written as $\underset{\text { Hocolim }}{\longrightarrow} Y_{n}$, where $Y_{n} \in T$.

Proof. Choose an augmentation for $Y$; that is, choose a basis for each $Y^{i}$. Let $Q_{i} \subset Y^{i}$ be the basis; each $Q_{i}$ is of cardinality $\leq \aleph_{0}$, that is finite or countable. By Lemma 5.7 there is a sequence of augmented subcomplexes

$$
Y_{0} \subset Y_{1} \subset Y_{2} \subset Y_{3} \subset \cdots
$$

such that:

(i) Each $Y_{n}$ is isomorphic to an object in $T$.

(ii) The union $\bigcup_{n=0}^{\infty} Y_{n}$ is an augmented subcomplex containing all the $Q_{i}$.

Since $Q_{i}$ spans $Y^{i}$, it follows that the union of the subcomplexes $Y_{n}$ is all of $Y$. But each $Y_{n}$ is isomorphic to an object in $T$, and $Y$ is the colimit. Moreover, it is a particularly nice colimit, as in Remark 6.3; hence the colimit $Y$ agrees with the homotopy colimit in $\mathbf{K}(R$-Proj).

To see this we look at a single degree $i$. We have a basis $Q_{i}$ for $Y^{i}$; we can write it as $Q_{i}=\left\{e_{\mu}, \mu \in M\right\}$. Then the sequence of modules

$$
Y_{0}^{i} \longrightarrow Y_{1}^{i} \longrightarrow Y_{2}^{i} \longrightarrow Y_{3}^{i} \longrightarrow \cdots
$$

is the direct sum of sequences

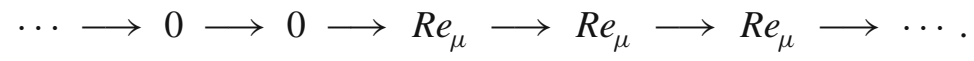

That is, for some of the $Y_{n}^{i}$ we have $e_{\mu} \notin Y_{n}^{i}$, and eventually it will be included. Since $Y$ is an augmented subcomplex, each $Y_{n}^{i}$ is the direct sum of the submodules $R e_{\mu}$ contained in it.

Proposition 7.4. The category $\mathbf{K}(R \text {-Proj })^{\aleph_{1}}$ consists precisely of the objects $Y$ isomorphic, in $\mathbf{K}(R$-Proj), to chain complexes of free modules of rank $\leq \aleph_{0}$. 
Proof. Lemma 7.2 proves that every object in $\mathbf{K}(R \text {-Proj })^{\aleph_{1}}$ is such a chain complex. For the reverse take a chain complex $Y$, of free modules of rank $\leq \aleph_{0}$. We will show that $Y$ lies in $\mathbf{K}(R \text {-Proj })^{\aleph_{1}}$.

By Lemma 7.3, $Y$ is isomorphic in $\mathbf{K}\left(R\right.$-Proj) to Hocolim $Y_{n}$, with $Y_{n} \in T$. Lemma 7.1 tells us that each $Y_{n}$ must lie in $\mathbf{K}(R \text {-Proj })^{\aleph_{1}}$. Now by [11, Lemma 4.2 .5$]$ the category $\mathbf{K}(R \text {-Proj })^{\aleph_{1}}$ is $\aleph_{1}$-localising, and by the above it contains all the $Y_{n}$. Hence it contains the homotopy colimit $Y$.

We do not need the following observation but include it in passing, just for general interest. Since the result will not be used, we will permit ourselves to leave to the reader some details of the proof.

Proposition 7.5. Let $\alpha>\aleph_{1}$ be a regular cardinal. I assert that the category $\mathbf{K}(R \text {-Proj })^{\alpha}$ consists precisely of all objects $Y$ isomorphic, in $\mathbf{K}(R$-Proj $)$, to chain complexes of free modules of rank $<\alpha$.

Proof. Let $\&$ be the full subcategory of $\mathbf{K}(R$-Proj), whose objects are all the isomorphs, in $\mathbf{K}(R$-Proj), of complexes of free modules of rank $<\alpha$. We need to show that $\mathbf{K}(R \text {-Proj })^{\alpha}=\&$. Clearly $\&$ is an $\alpha$-localizing subcategory of $\mathbf{K}(R$-Proj $)$. It contains $T$, which is contained in $\mathbf{K}(R \text {-Proj })^{\aleph_{1}} \subset$ $\mathbf{K}(R \text {-Proj })^{\alpha}$. Since $T$ generates $\mathbf{K}(R$-Proj $)$, [11, Lemma 4.4.5] applies and tells us that $\mathbf{K}(R \text {-Proj })^{\alpha} \subset \&$.

We need to prove the reverse inclusion $\& \subset \mathbf{K}(R \text {-Proj })^{\alpha}$. For this it suffices to show that $\delta$ is an $\alpha$-perfect class of $\alpha$-small objects, and hence contained in $\mathbf{K}(R \text {-Proj })^{\alpha}$, which is the maximal such class. If $X$ is an object of $\delta$, we need to show that any map

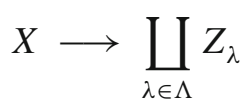

factors as

$$
X \longrightarrow \coprod_{\lambda \in \Lambda^{\prime}} Y_{\lambda} \stackrel{\bigsqcup_{\lambda \in \Lambda^{\prime}} f_{\lambda}}{\longrightarrow} \coprod_{\lambda \in \Lambda^{\prime}} Z_{\lambda} \stackrel{\text { inclusion }}{\longrightarrow} \coprod_{\lambda \in \Lambda} Z_{\lambda}
$$

where $\Lambda^{\prime} \subset \Lambda$ is a subset of cardinality $<\alpha$, and $f_{\lambda}: Y_{\lambda} \longrightarrow Z_{\lambda}$ are some morphisms with $Y_{\lambda} \in$ \&.

One begins by replacing each $Z_{\lambda}$ by an augmented complex, up to isomorphism in $\mathbf{K}(R$-Proj); see Lemma 5.3. Because $X$ belongs to $\&$, we may replace it by an isomorphic augmented complex where each $X^{i}$ is free of rank $<\alpha$. Choose a chain map

$$
X \longrightarrow \coprod_{\lambda \in \Lambda} Z_{\lambda}
$$

representing the homotopy equivalence class we are given. With these replacements made, we assert that our desired factorization may already be 
made in the category $\mathbf{C}(R$-Proj $)$, and that the $Y_{\lambda}$ may be chosen to be augmented subcomplexes of $Z_{\lambda}$. To do this, observe that every element of the basis of $X$ maps to a finite linear combination of basis elements of the $Z_{\lambda}$. For each $x$ in the basis of $X$ choose such a finite set $F_{x}$ in the union of bases of the $Z_{\lambda}$. The union of the $F_{x}$, over all $x$ in the basis for $X$, is a set $F$ of cardinality $<\alpha$. Let $\Lambda^{\prime} \subset \Lambda$ be the set of all $\lambda$ for which $F \cap Z_{\lambda} \neq \emptyset$; clearly the cardinality $\Lambda^{\prime}$ is $<\alpha$. Also, the cardinality of $F \cap Z_{\lambda}$ is $<\alpha$. The part we leave to the reader is to show that we may choose an augmented subcomplex $Y_{\lambda} \subset Z_{\lambda}$ containing $F \cap Z_{\lambda}$, and where the basis for each $Y_{\lambda}^{i}$ has cardinality $<\alpha$. The hint is to generalize the proofs of Lemmas 5.5 and 5.7, which did the countable case.

Proposition 7.4 gives us a complete description of the $\aleph_{1}$-compact objects in $\mathbf{K}(R$-Proj). In Proposition 7.5 we noted, in passing, what are the $\alpha$-compact objects for $\alpha>\aleph_{1}$. Now we would like to understand the $\aleph_{0}$-compact objects, known more simply as just the compact objects.

Lemma 7.6. Every compact object is isomorphic to an object in $T$.

Proof. Let $X$ be a compact object in $\mathbf{K}(R$-Proj). By [11, Lemma 4.2.3] we know that $\mathbf{K}(R \text {-Proj })^{\aleph_{0}} \subset \mathbf{K}(R \text {-Proj })^{\aleph_{1}}$; that is, all $\aleph_{0}$-compact objects are $\aleph_{1}$-compact. We know the $\aleph_{1}$-compact objects. $X$ must be isomorphic, in $\mathbf{K}\left(R\right.$-Proj), to a chain complex $Y$ of free modules of rank $\leq \aleph_{0}$.

Lemma 7.3 says that $Y$ is a homotopy colimit of objects $Y_{n} \in T$. By [10, Lemma 2.8], the isomorphism $X \longrightarrow Y=$ Hocolim $Y_{n}$ must factor through some $Y_{n}$. We conclude that $X$ must be a direct summand of some $Y_{n}$. To complete the proof it suffices to show that every direct summand of an object $Y_{n} \in T$ is homotopy equivalent to an object in $T$; this follows from [3, Proposition 3.4] applied to the complex $Y_{n}^{*} \in \mathbf{K}^{-}\left(R^{\text {op }}\right.$-proj), with $Y_{n}^{*}$ as in Definition 7.7 below.

In the proof of Lemma 7.6 we met the complexes $Y^{*}$, without being formally introduced; it is time to rectify this. The complexes $Y^{*}$ will play a key role in what follows; they will permit us to identify which objects of $T$ are compact.

Definition 7.7. Let $Y$ be an object of $\mathbf{K}(R$-proj); that is $Y$ is a complex

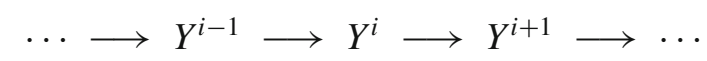

of finitely generated, projective $R$-modules. The complex $Y^{*}=\mathscr{H} \mathrm{om}(Y, R)$ is the dual; it is the complex of right $R$-modules

$$
\cdots \rightarrow \operatorname{Hom}\left(Y^{i+1}, R\right)^{-i-1} \rightarrow \operatorname{Hom}\left(Y^{i}, R\right)^{-i} \rightarrow \operatorname{Hom}\left(Y^{i-1}, R\right)^{-i+1} \rightarrow \cdots
$$

The notation is intended to indicate that this is the chain complex, in which the module $\operatorname{Hom}\left(Y^{i}, R\right)$ is placed in degree $-i$. 
Remark 7.8. The complex $Y^{*}$ is an object in $\mathbf{K}\left(R^{\text {op }}\right.$-proj $)$; it is a chain complex of finitely generated, projective right $R$-modules. If $Y^{i}$ vanishes when $i \ll 0$, then $\left\{Y^{*}\right\}^{i}$ vanishes when $i \gg 0$. A map $f: X \longrightarrow Y$ in $\mathbf{K}(R$-proj $)$ vanishes if and only if the dual $f^{*}: Y^{*} \longrightarrow X^{*}$ vanishes in $\mathbf{K}\left(R^{\text {op }}\right.$-proj $)$; the dual of a homotopy of $f$ with the null map is a homotopy of $f^{*}$ with the null map.

Lemma 7.9. If an object $Y \in T$ is compact, then $H^{i}\left(Y^{*}\right)=0$ for all $i \ll 0$.

Proof. We prove the contrapositive; that is, we will assume $H^{i}\left(Y^{*}\right) \neq 0$ for infinitely many $i$, and deduce that $Y$ cannot be compact. Suppose $H^{i}\left(Y^{*}\right) \neq 0$ for infinitely many $i$. This means that, for infinitely many $i \in \mathbb{Z}$, we can choose a non-zero element in $H^{i}\left(Y^{*}\right)$. Any such element corresponds to a map $\Sigma^{-i} R^{\text {op }} \longrightarrow Y^{*}$, not homotopic to zero. Dually we have infinitely many maps, all not homotopic to zero, $Y \longrightarrow \Sigma^{i} R$. These assemble to a map $Y \longrightarrow \prod_{i} \Sigma^{i} R$. Since the $\Sigma^{i} R$ are all in different degrees, this factors as

$$
Y \longrightarrow \coprod_{i} \Sigma^{i} R \stackrel{\simeq}{\longrightarrow} \prod_{i} \Sigma^{i} R
$$

where the map from the coproduct to the product is an isomorphism. This map visibly does not factor through a finite direct sum, and we conclude that $Y$ cannot be compact.

Lemma 7.10. Suppose $Y \in T$, and suppose $H^{i}\left(Y^{*}\right)=0$ for all $i \ll 0$. Then there exists an integer $n \in \mathbb{Z}$, depending on $Y$, satisfying the following.

(i) If $Z \in T$, and $Z^{i}=0$ for all $i<n$, then any map $Y \longrightarrow Z$ is null homotopic.

Proof. We are given that $H^{i}\left(Y^{*}\right)=0$ for all $i \ll 0$. This means that there exists an integer $n \in \mathbb{N}$, so that $H^{i}\left(Y^{*}\right)=0$ for all $i \leq-n$. We assert that this $n$ works; it satisfies the assertion of the Lemma.

Suppose $Z \in T$, and $Z^{i}=0$ for all $i<n$; we need to prove that any map $Y \longrightarrow Z$ is null homotopic. Because $Z^{i}=0$ for all $i<n$, we know that $\left\{Z^{*}\right\}^{i}=0$ for all $i>-n$. Now $Z^{*}$ is a bounded above complex of projectives; more explicitly, it vanishes in degrees $>-n$. For a bounded above chain complex $Z^{*}$ of projective $R^{\mathrm{op}}$-modules, there is a $1-1$ correspondence

$$
\operatorname{Hom}_{\mathbf{K}\left(R^{\text {op-Mod })}\right.}\left(Z^{*}, Y^{*}\right)=\operatorname{Hom}_{\mathbf{D}\left(R^{\text {op-Mod })}\right.}\left(Z^{*}, Y^{*}\right) ;
$$

that is, maps in the derived category correspond bijectively with homotopy equivalence classes of chain maps. But in the derived category $\mathcal{T}=$ $\mathbf{D}\left(R^{\mathrm{op}}-\mathrm{Mod}\right)$ there is a standard $t$-structure, and $Z^{*}$ lies in $\mathcal{T}^{\leq-n}$ while $Y^{*}$ lies in $\mathcal{T}^{>-n}$. Hence

$$
\begin{aligned}
\operatorname{Hom}_{\mathbf{K}(R-\text { Mod })}(Y, Z) & =\operatorname{Hom}_{\mathbf{K}\left(R^{\text {op-Mod }}\right)}\left(Z^{*}, Y^{*}\right) \\
& =\operatorname{Hom}_{\mathbf{D}\left(R^{\text {op-Mod }}\right)}\left(Z^{*}, Y^{*}\right)=0 .
\end{aligned}
$$


Lemma 7.11. Suppose $Y \in T$, and suppose $H^{i}\left(Y^{*}\right)=0$ for all $i \ll 0$. Then $Y$ is a compact object of $\mathbf{K}(R$-Flat).

Proof. By Lemma 7.10 there exists an integer $n \in \mathbb{Z}$, so that, if $Z \in T$ and $Z^{i}=0$ for $i<n$, then any map $Y \longrightarrow Z$ is null homotopic. Choose and fix such an $n$.

Because $Y$ is in $T$, Lemma 4.5 tells us that $Y$ is $\aleph_{1}$-small. Any map from $Y$, into an arbitrary coproduct, factors through a coproduct of $\leq \aleph_{0}$ objects. It remains, therefore, to show that any map

$$
Y \longrightarrow \coprod_{\lambda=1}^{\infty} Z_{\lambda}
$$

factors through a finite coproduct. Now Lemma 4.6 tells us that there is a factorization

$$
Y \longrightarrow \coprod_{\lambda=1}^{\infty} Y_{\lambda} \longrightarrow \coprod_{\lambda=1}^{\infty} Z_{\lambda}
$$

with $Y_{\lambda} \in T$. Refinement 4.8 tells us, more precisely, that all but finitely many of the $\left\{Y_{\lambda}^{i}, i<n\right\}$ vanish. Except for finitely many $\lambda$, the modules $Y_{\lambda}^{i}$ vanish if $i<n$. This means that, except for finitely many $\lambda$, the maps $Y \longrightarrow Y_{\lambda}$ are null homotopic. Hence the map $Y \longrightarrow \bigsqcup_{\lambda=1}^{\infty} Y_{\lambda}$ factors, up to homotopy, through a finite sum. The reader should note that, in assembling a bunch of homotopies $Y \Longrightarrow Y_{\lambda}$ into a single homotopy $Y \Longrightarrow \bigsqcup_{\lambda} Y_{\lambda}$, we are again using Refinement 4.8; we use the fact that, in each degree $i$, all but finitely many $Y_{\lambda}^{i}$ vanish.

Putting it all together, we have

Proposition 7.12. An object of $\mathbf{K}(R$-Proj) is compact if and only if it is isomorphic, in $\mathbf{K}(R$-Proj), to a complex $Y$ satisfying

(i) $Y$ is a complex of finitely generated projective modules.

(ii) $Y^{i}=0$ if $i \ll 0$.

(iii) $H^{i}\left(Y^{*}\right)=0$ if $i \ll 0$.

Proof. Assertions (i) and (ii) say that $Y$ is isomorphic to an object in $T$. In other words, the compact objects are, up to isomorphism in $\mathbf{K}(R$-Proj), the objects in $T$ satisfying (iii).

The necessity was proved in Lemmas 7.6 and 7.9; a compact object must satisfy (i)-(iii). For the sufficiency, Lemma 7.11 showed that an object satisfying (i)-(iii) is compact not only in $\mathbf{K}(R$-Proj), but also in the larger $\mathbf{K}(R$-Flat $)$.

Remark 7.13. We noted, in the last line of the proof of Proposition 7.12, that the sequence of lemmas proved a little more than Proposition 7.12 asserted. Proposition 7.12 identifies for us precisely what are the compact objects of $\mathbf{K}(R$-Proj); up to isomorphism, they are the objects $Y \in T$, 
with $H^{i}\left(Y^{*}\right)=0$ for $i \ll 0$. But Lemma 7.11 established more; it proved that the objects satisfying this condition are compact in the larger category $\mathbf{K}(R$-Flat $)$. In other words, in the course of the proof we also showed that the inclusion functor $j_{1}: \mathbf{K}(R$-Proj $) \longrightarrow \mathbf{K}(R$-Flat $)$ takes compacts to compacts. This has implications, to which we will return in Remark 8.2.

Proposition 7.12 tells us what are the compact objects in $\mathbf{K}(R$-Proj); the obvious next question is to determine when they generate. We do not understand this fully, but in the remainder of the section we will discuss what we know.

Proposition 7.14. If $R^{\mathrm{op}}$ is a coherent ring, then the category $\mathbf{K}(R$-Proj) is compactly generated.

Proof. Corollary 5.10 tells us that the set $T$ generates the category $\mathbf{K}(R$-Proj). It therefore suffices to show that any localizing subcategory containing $\mathbf{K}(R \text {-Proj })^{\aleph_{0}}$ contains $T$.

Let $Y$ be an object in $T$. Replacing $Y$ by a suitable suspension, we may assume that $Y$ is a chain complex of finitely generated projective modules

$$
\cdots \longrightarrow 0 \longrightarrow 0 \longrightarrow Y^{0} \longrightarrow Y^{1} \longrightarrow Y^{2} \longrightarrow \cdots .
$$

This makes $Y^{*}$ the chain complex

$$
\cdots \longrightarrow\left\{Y^{2}\right\}^{*} \stackrel{\partial_{-2}}{\longrightarrow}\left\{Y^{1}\right\}^{*} \stackrel{\partial_{-1}}{\longrightarrow}\left\{Y^{0}\right\}^{*} \longrightarrow 0 \longrightarrow 0 \longrightarrow \cdots .
$$

Now consider the $t$-structure truncations $\left\{Y^{*}\right\}^{\geq-i}$; these are, up to isomorphism in the derived category, the complexes

$$
\cdots \longrightarrow 0 \longrightarrow 0 \longrightarrow \frac{\left\{Y^{i}\right\}^{*}}{\operatorname{Im}\left(\partial_{-i-1}\right)} \longrightarrow\left\{Y^{i-1}\right\}^{*} \longrightarrow\left\{Y^{i-2}\right\}^{*} \longrightarrow \cdots
$$

Since the map $\partial_{-i-1}:\left\{Y^{i+1}\right\}^{*} \longrightarrow\left\{Y^{i}\right\}^{*}$ is a map of finitely generated, projective $R^{\mathrm{op}}$-modules, and since the ring $R^{\mathrm{op}}$ is assumed coherent, the module $\operatorname{Im}\left(\partial_{-i-1}\right)$ has a resolution by finitely generated projective modules. There exists a complex

$$
\longrightarrow Z_{i}^{-i-3} \longrightarrow Z_{i}^{-i-2} \longrightarrow Z_{i}^{-i-1} \longrightarrow\left\{Y^{i}\right\}^{*} \longrightarrow\left\{Y^{i-1}\right\}^{*} \longrightarrow
$$

of finitely generated, projective $R^{\mathrm{op}}$-modules, which is isomorphic in the derived category $\mathbf{D}\left(R^{\text {op }}\right.$-Mod $)$ to $\left\{Y^{*}\right\}^{\geq-i}$. Choose such a complex, and call it $Z_{i}$. All the complexes $Z_{i}$ are bounded above complexes of projectives, and hence maps in the derived category correspond bijectively to homotopy equivalence classes of chain maps. It follows that we can choose 
chain maps $Z_{i+1} \longrightarrow Z_{i}$, lifting the canonical map in the derived category $\left\{Y^{*}\right\}^{\geq-i-1} \longrightarrow\left\{Y^{*}\right\}^{\geq-i}$. That is, there is a chain map

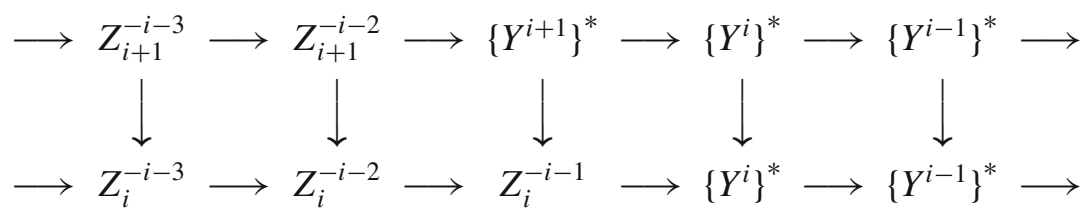

lifting the map of $t$-structure truncations. We leave it to the reader to check that this chain map can be chosen so that, in degrees $j \leq i$, it is just the identity map $1:\left\{Y^{j}\right\}^{*} \longrightarrow\left\{Y^{j}\right\}^{*}$. That is, the diagram above becomes

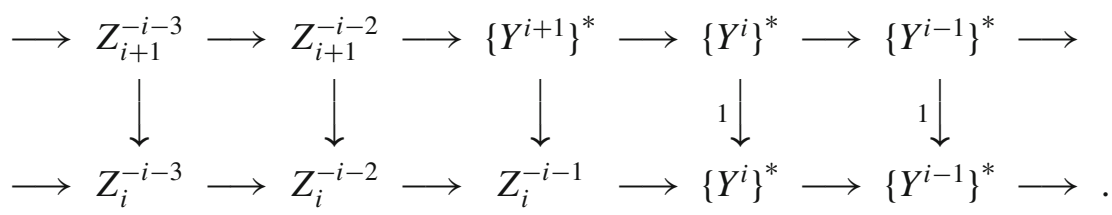

Dualizing, we have a sequence of chain maps

$$
Z_{0}^{*} \longrightarrow Z_{1}^{*} \longrightarrow Z_{2}^{*} \longrightarrow Z_{3}^{*} \longrightarrow \cdots .
$$

The $Z_{n}^{*}$ are compact objects in $\mathbf{K}(R$-Proj) by construction, and $Y$ is the colimit. Since in each degree $i$ the sequence $\left\{Z_{n}^{*}\right\}^{i}$ eventually stabilizes to $Y^{i}$, Remark 6.3 applies. The chain complex $Y$ is the homotopy colimit of the complexes $Z_{n}^{*}$.

Remark 7.15. In Propostion 7.14 we saw that, if the ring $R^{\text {op }}$ is coherent, then the triangulated category $\mathbf{K}(R$-Proj $)$ is compactly generated. The coherence of $R^{\mathrm{op}}$ is not necessary. Another example of a class of rings $R$, for which $\mathbf{K}(R$-Proj) is compactly generated, are the rings of finite global dimension. We remind the reader that a ring $R$ has finite global dimension if there exists an integer $n \in \mathbb{N}$, so that every $R$-module has a projective resolution of length $\leq n$. For rings of finite global dimension, the homotopy category $\mathbf{K}(R$-Proj) is equivalent to the derived category $\mathbf{D}(R$-Mod $)$, which is always compactly generated.

It therefore becomes interesting to see an example of a ring $R$, for which the category $\mathbf{K}(R$-Proj) is not compactly generated. We end this section by giving such an example.

Example 7.16. Let $k$ be a field, and let $\mathfrak{m}$ be an infinite dimensional vector space over $k$. Let $R$ be the ring $k \oplus \mathfrak{m}$; the addition is obvious, and the multiplication is the one that makes $\mathfrak{m}$ into a square-zero ideal; that is, for all $a, a^{\prime} \in k$ and $b, b^{\prime} \in \mathfrak{m}$, we define

$$
(a \oplus b) \cdot\left(a^{\prime} \oplus b^{\prime}\right)=\left(a a^{\prime} \oplus\left(a^{\prime} b+a b^{\prime}\right)\right) .
$$

I assert that the category $\mathbf{K}(R$-Proj $)$ is not compactly generated. 
Let us sketch the proof. To begin with, observe that all projective $R$-modules are free. Also, any chain complex $Y$ of projective (= free) $R$-modules can be decomposed as $Y=X \oplus X^{\prime}$, with $X^{\prime}$ contractible, and so that all the differentials in the chain complex $X$ vanish modulo the ideal $\mathfrak{m}$. Maybe we should briefly remind the reader of the proof of the second fact.

Let $Y$ be a chain complex of free $R$-modules. Form the complex $k \otimes_{R} Y$; it is a complex of $k$-vector spaces. Let $I$ be the image of the map $k \otimes Y^{i} \longrightarrow$ $k \otimes Y^{i+1}$. The map $k \otimes Y^{i} \longrightarrow I$ is a surjective map of vector spaces, and hence admits a splitting. Choose one; call it $i: I \longrightarrow k \otimes Y^{i}$. Choose a basis for the image of $I \longrightarrow k \otimes Y^{i}=Y^{i} / \mathfrak{m} Y^{i}$, and then choose elements of $Y^{i}$ which reduce, modulo $\mathfrak{m} Y^{i}$, to your chosen basis. This produces, for each $i$, a map $F_{i} \longrightarrow Y^{i}$, where $F_{i}$ is a free module. We leave it to the reader to check that the chain map

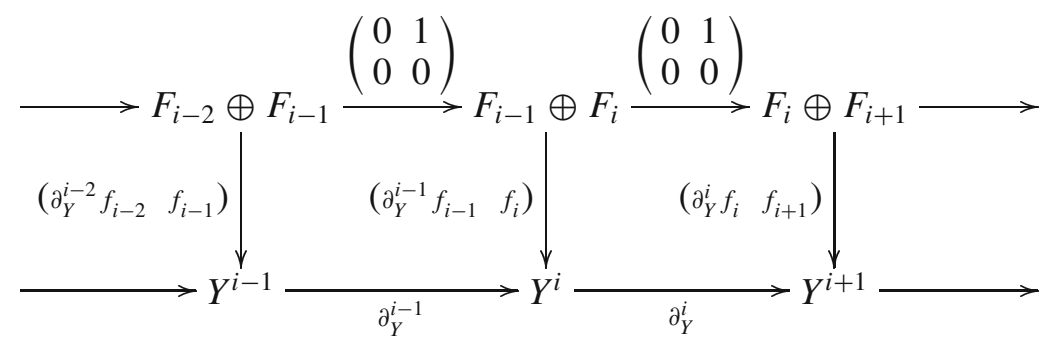

is split injective in each degree. Call this chain map $f: X^{\prime} \longrightarrow Y$. If we complete to a short exact sequence of chain complexes

$$
0 \longrightarrow X^{\prime} \longrightarrow Y \longrightarrow X \longrightarrow 0,
$$

then in each degree this sequence is split; $Y$ must be the mapping cone on some chain map $\Sigma^{-1} X \longrightarrow X^{\prime}$. But $X^{\prime}$ is contractible, so any chain map $\Sigma^{-1} X \longrightarrow X^{\prime}$ is null homotopic. Hence $Y$ is isomorphic to $X \oplus X^{\prime}$. The fact that the differentials in the chain complex $X$ vanish, modulo the ideal $\mathfrak{m}$, is immediate once we tensor the short exact sequence of chain complexes with $k=R / \mathfrak{m}$.

Now Proposition 7.12 characterizes for us the compact objects in $\mathbf{K}(R$-Proj); they are isomorphic, in $\mathbf{K}(R$-Proj), to complexes $Y$ of finitely generated, projective $R$-modules, with $Y^{i}=0=H^{i}\left(Y^{*}\right)$ if $i \ll 0$. By the above $Y=X \oplus X^{\prime}$, with $X^{\prime}$ contractible and $X$ having differentials that vanish modulo the ideal $\mathfrak{m}$. Replacing $Y$ by $X$ (the two are isomorphic in $\mathbf{K}(R$-Proj)) we may assume that the differentials in the chain complex $Y$ vanish modulo $\mathfrak{m}$. This means that the differential of $Y^{*}$ must also vanish modulo $\mathfrak{m}$. We have that $Y^{*}$ is a complex of finitely generated, free $R$-modules, so that

(i) The differentials $\partial^{i}:\left\{Y^{*}\right\}^{i} \longrightarrow\left\{Y^{*}\right\}^{i+1}$ vanish modulo the ideal $\mathfrak{m}$. That is, they are given by matrices with entries in $\mathfrak{m}$.

(ii) $H^{i}\left(Y^{*}\right)=0$ if $i \ll 0$.

(iii) $\left\{Y^{*}\right\}^{i}=\left\{Y^{-i}\right\}^{*}=0$ if $i \gg 0$. 
From Condition (i) it follows that the image of $\partial^{i}:\left\{Y^{*}\right\}^{i} \longrightarrow\left\{Y^{*}\right\}^{i+1}$ is a finite dimensional vector space over $k$; it is a quotient of the finite dimensional $k \otimes\left\{Y^{*}\right\}^{i}$. On the other hand the kernel must contain $\mathfrak{m}\left\{Y^{*}\right\}^{i}$; if $\left\{Y^{*}\right\}^{i}$ is non-zero, this kernel is infinite dimensional. We deduce that $H^{i}\left(Y^{*}\right)$ can only vanish if $\left\{Y^{*}\right\}^{i}=0$. Thus the complex $Y^{*}$ must satisfy $\left\{Y^{*}\right\}^{i}=0$ if either $i \gg 0$ or $i \ll 0$; it is a bounded complex of projective $R$-modules. Hence so is the dual $Y$; the compact objects of $\mathbf{K}(R$-Proj), for our particular $R$, are the bounded complexes of finitely generated projective modules.

Let $Y$ be a compact object in $\mathbf{K}(R$-Proj). For any acyclic complex $Z$, any map $Y \longrightarrow Z$ must be null homotopic; maps from bounded above complexes of projectives to acyclic complexes are null homotopic. To show that $\mathbf{K}(R$-Proj $)$ is not compactly generated, it therefore suffices to exhibit an acyclic complex of free modules, which is not null homotopic.

Let $M$ be the module

$$
M=R \otimes_{k} \bigotimes_{i=1}^{\infty} \mathfrak{m} .
$$

We define a map $\varphi: M \longrightarrow M$ by the formula

$$
\varphi\left(r \otimes b_{1} \otimes b_{2} \otimes b_{3} \otimes \cdots\right)=\left(r b_{1} \otimes b_{2} \otimes b_{3} \otimes \cdots\right) .
$$

The complex

$$
\text { ․ } \stackrel{\varphi}{\longrightarrow} M \stackrel{\varphi}{\longrightarrow} M \stackrel{\varphi}{\longrightarrow} M \stackrel{\varphi}{\longrightarrow} \cdots
$$

is clearly acyclic, but cannot be null homotopic. Any map $\Theta^{i+1} \varphi+\varphi \Theta^{i}$ must vanish modulo $\mathfrak{m}$; it cannot be the identity.

\section{The right adjoint to $j_{!}: \mathbf{K}(R$-Proj $) \longrightarrow \mathbf{K}(R$-Flat $)$}

We have an inclusion of categories $j_{!}: \mathbf{K}(R$-Proj $) \longrightarrow \mathbf{K}(R$-Flat $)$. In this section we will study the right adjoint to this inclusion. In particular we will identify the subcategory $\mathbf{K}(R \text {-Proj })^{\perp} \subset \mathbf{K}$ (R-Flat), on which this right adjoint vanishes.

Proposition 8.1. The natural inclusion $j_{!}: \mathbf{K}(R$-Proj $) \longrightarrow \mathbf{K}(R$-Flat $)$ has a right adjoint $j^{*}: \mathbf{K}(R$-Flat $) \longrightarrow \mathbf{K}(R$-Proj $)$.

Proof. The functor $j_{\text {! }}$ is triangulated and respects coproducts, while Corollary 5.10 tells us that the category $\mathbf{K}(R$-Proj) satisfies Brown representability. The proposition now follows from [11, Theorem 8.4.4].

Remark 8.2. We already know a little about the functor $j^{*}$; it is easy to show that it respects coproducts. We will, in a sequel to this article, prove rather more: we will show that the functor $j^{*}$ always has a right adjoint. Let us nevertheless discuss why we already know as much as we do. 
Let us begin the discussion by assuming that the category $\mathbf{K}(R$-Proj) happens to be compactly generated. In Remark 7.13 we noted that the inclusion functor $j_{1}: \mathbf{K}(R$-Proj $) \longrightarrow \mathbf{K}(R$-Flat $)$ takes compact objects in $\mathbf{K}(R$-Proj) to compact objects in $\mathbf{K}(R$-Flat). From [10, Theorem 5.1] it now follows that the functor $j^{*}$ respects coproducts.

If $R$ is general, in which case $\mathbf{K}(R$-Proj) need not be compactly generated, we need another argument. Theorem 5.9 tells us that $T$ is an $\aleph_{1}$-compact generating set for $\mathbf{K}(R$-Proj). Next observe that Lemmas 4.5 and 4.6 assert, in our present notation, that the inclusion functor $j_{!}: \mathbf{K}(R$-Proj $) \longrightarrow$ $\mathbf{K}(R$-Flat $)$ takes $T \subset \mathbf{K}(R$-Proj $)$ to an $\aleph_{1}$-perfect set of $\aleph_{1}$-small objects in $\mathbf{K}(R$-Flat $)$. We assert that this is enough; it formally follows that the functor $j^{*}$ respects coproducts. For this we need an $\alpha$-generalization of [10, Theorem 5.1]; we include it in Appendix A, for the reader's entertainment.

The natural inclusion provides a fully faithful functor of triangulated categories $j_{1}: \mathbf{K}(R$-Proj $) \longrightarrow \mathbf{K}(R$-Flat $)$, and Proposition 8.1 guarantees that it has a right adjoint. The general nonsense formalism of Remark 2.12 applies, and it becomes interesting to study the category $\mathbf{K}(R \text {-Proj })^{\perp}$. In the next few lemmas we will figure out what it is.

Reminder 8.3. Let $\mathcal{T}$ be a category, $z \in \mathcal{T}$ an object, and $T \subset \mathcal{T}$ a set of objects. We remind the reader of the comma category $T / z$.

The objects of $T / z$ are morphisms $f: t \longrightarrow z$, where $t \in T$. A morphism in $T / z$, from the object $f: t \longrightarrow z$ to the object $f^{\prime}: t^{\prime} \longrightarrow z$, is a commutative triangle

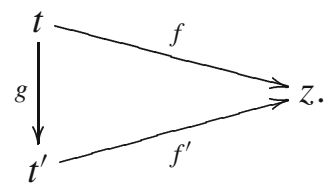

There is a functor $F: T / z \longrightarrow \mathcal{T}$, taking the object $f: t \longrightarrow z$ to the object $t$.

Note that $T$ is assumed to be a set of objects, forcing $T / z$ to be a small category. If small colimits exist in $\mathcal{T}$, then the functor $F: T / z \longrightarrow \mathcal{T}$ must have a colimit. The colimit of $F$ has a natural map to $z$.

Lemma 8.4. In Reminder 8.3 consider the special case where $\mathcal{T}=$ $\mathbf{C}(R$-Mod $), T$ is the set of Construction 4.3 , and $z$ lies in $\mathbf{C}(R$-Flat $) \subset$ $\mathbf{C}(R$-Mod). We will establish two things:

(i) The map colim $F \longrightarrow z$ is an isomorphism.

(ii) The category $T / z$ is filtered.

Proof. Let us begin by proving (i). We need to show that the map $\underset{\text { colim }}{\longrightarrow} \longrightarrow z$ is injective and surjective; we first prove surjectivity. Let 
$z$ be the chain complex

$$
\cdots \longrightarrow Z^{i-1} \longrightarrow Z^{i} \longrightarrow Z^{i+1} \longrightarrow Z^{i+2} \longrightarrow \cdots
$$

and let $x \in Z^{i}$ be any element. We need to show that $x$ is in the image of the map colim $F \longrightarrow z$. To do this, choose any map $f^{i}: R \longrightarrow Z^{i}$, with $x$ in its image. Consider the chain map

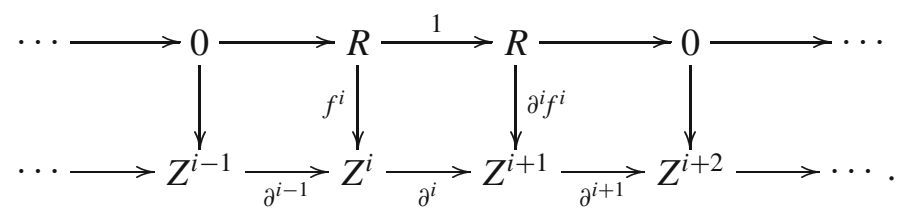

The top row is a chain complex $r$ belonging to the set $T$ of Construction 4.3. The chain map is therefore an object $f: r \longrightarrow z$ in the category $T / z$. It follows that the map $f: r \longrightarrow z$ factors as $r \longrightarrow \underset{\operatorname{colim}}{\longrightarrow} \longrightarrow z$; since $x \in Z^{i}$ lies in the image of the composite, it must lie in the image of $\underset{\operatorname{colim}}{\longrightarrow} F \longrightarrow z$.

Next we prove the map colim $F \longrightarrow z$ injective. Suppose $f: s \longrightarrow z$ and $g: t \longrightarrow z$ are two objects of $T / z$, and suppose some element of $Z^{i}$ lies in the image of both. This means that we have two chain maps of chain complexes

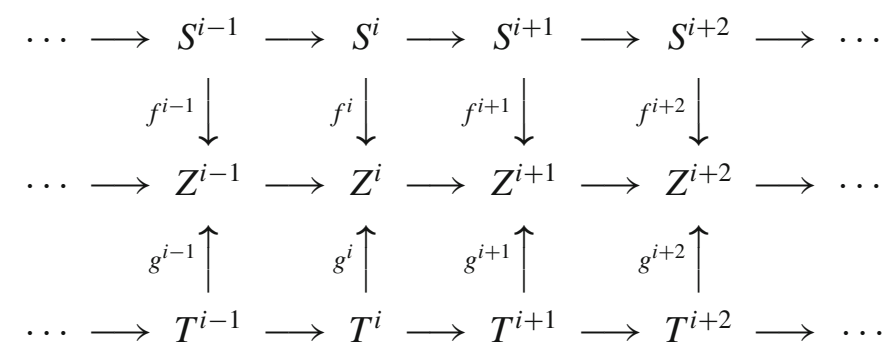

as well as elements $x \in S^{i}$ and $x^{\prime} \in T^{i}$, with $f^{i}(x)=g^{i}\left(x^{\prime}\right)$. Let $\rho^{i}$ : $R \longrightarrow S^{i}$ be the $R$-module homomorphism with $\rho^{i}(1)=x$, and let $\sigma^{i}$ : $R \longrightarrow T^{i}$ be the $R$-module homomorphism with $\sigma^{i}(1)=x^{\prime}$. We can consider the chain maps

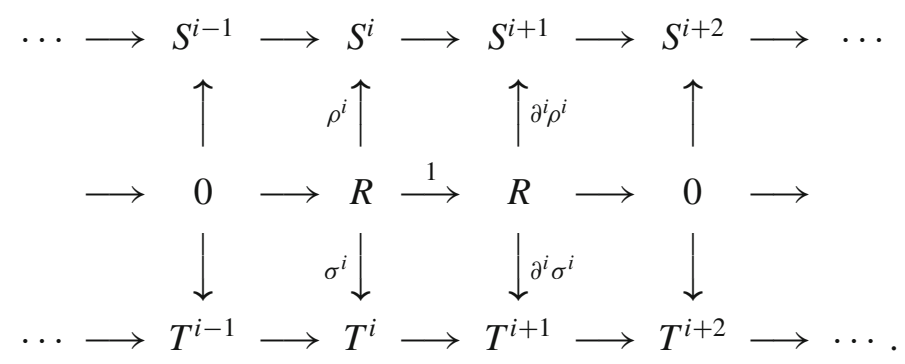


These chain maps yield a commutative diagram

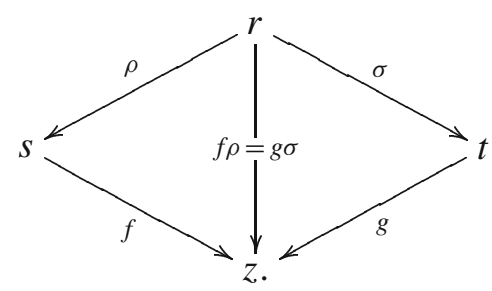

This diagram may be viewed as giving maps in $T / z$ from the object $r \longrightarrow z$ to the two objects $f: s \longrightarrow z$ and $g: t \longrightarrow z$. Hence the diagram below must commute

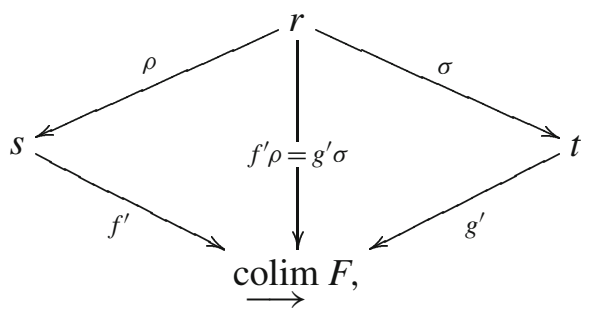

and it follows that the image in colim $F$ of $x \in S^{i}$ must agree with the image in $\operatorname{colim} F$ of $x^{\prime} \in T^{i}$.

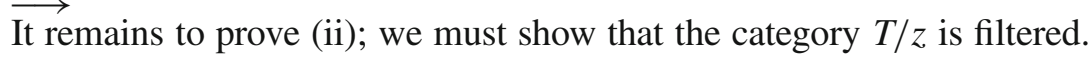
Given any two objects $f: s \longrightarrow z$ and $g: t \longrightarrow z$ in the category $T / z$, they map to a common object as below

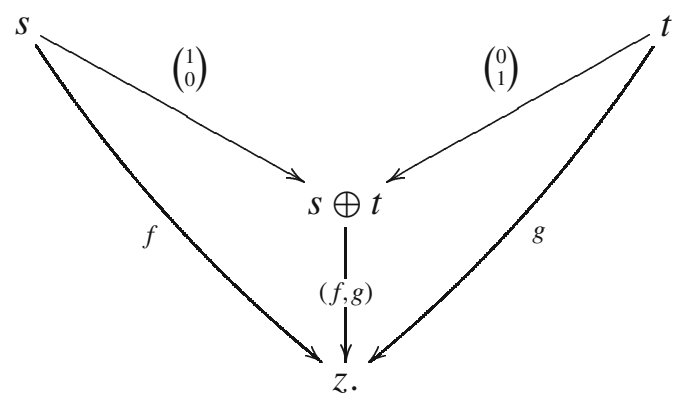

What needs proof is that, given two maps in $T / z$ from the object $f: s \longrightarrow z$ to the object $g: t \longrightarrow z$, they can be coequalized. Our two maps in $T / z$ amount to two commutative triangles
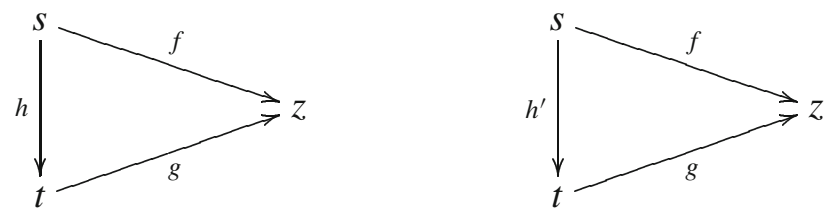
and hence the composite

$$
s \stackrel{h-h^{\prime}}{\longrightarrow} t \stackrel{g}{\longrightarrow} z
$$

must vanish. But $s$ and $t$ are objects in the set $T$ of Construction 4.3, while $z$ is a complex of flat $R$-modules. Lemma 4.2 applies. We deduce from Lemma 4.2 that the map $g: t \longrightarrow z$ factors as $t \stackrel{\rho}{\longrightarrow} w \stackrel{\sigma}{\longrightarrow} z$, with $w \in T$, and so that the composite

$$
s \stackrel{h-h^{\prime}}{\longrightarrow} t \stackrel{\rho}{\longrightarrow} w
$$

vanishes. The commutative triangle

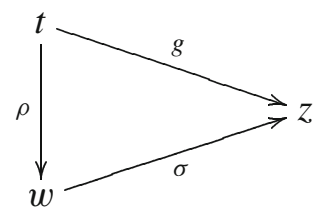

is a morphism in $T / z$ from the object $g: t \longrightarrow z$ to the object $\sigma: w \longrightarrow z$, coequalizing $h$ and $h^{\prime}$.

Lemma 8.5. Let $z$ be an object in the category $\mathbf{K}(R \text {-Proj })^{\perp}$. We remind the reader of the notation of Remark 2.12: this means that $z \in \mathbf{K}(R$-Flat) is such that any map, from an object $s \in \mathbf{K}(R$-Proj) to $z$, must be null homotopic. Then the chain complex $z$ can be expressed, in the category $\mathbf{C}(R-\mathrm{Mod})$, as a filtered colimit of contractible chain complexes of finitely generated, projective $R$-modules.

Proof. We know that $z \in \mathbf{K}(R$-Flat) is a complex of flat $R$-modules. Lemma 8.4 therefore tells us that $z$ is the colimit of the forgetful functor $F$ from the comma category $T / z$, and that $T / z$ is filtered.

The objects in the category $T / z$ are morphisms $f: t \longrightarrow z$, where $t$ is an object in $T \subset \mathbf{K}(R$-Proj). The hypothesis of the Lemma asserts that any map $t \longrightarrow z$ must be null homotopic. Hence the map $f: t \longrightarrow z$ must factor as

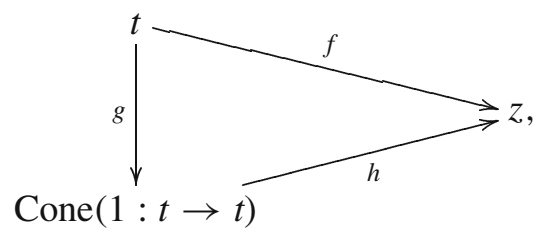

where Cone $(1: t \rightarrow t)$ is the mapping cone on the identity $1: t \longrightarrow t$. The complex Cone $(1: t \rightarrow t)$ is contractible and lies in $T$. The factorization above establishes that the objects $h: c \longrightarrow z$, with $c \in T$ a contractible complex, form a cofinal subcategory of $T / z$. Hence $z$ is a filtered direct limit of contractible complexes of finitely generated, projective $R$-modules. 
Theorem 8.6. Let $R$ be any ring, and let $Z$ be an object of $\mathbf{K}(R$-Flat). The following are equivalent:

(i) $Z$ lies in the subcategory $\mathbf{K}(R \text {-Proj })^{\perp} \subset \mathbf{K}(R$-Flat).

(ii) $Z$ is a filtered direct limit of contractible chain complexes of projective $R$-modules.

(iii) $Z$ is an acyclic complex of flat $R$-modules

$$
\cdots \longrightarrow Z^{i-1} \stackrel{\partial^{i-1}}{\longrightarrow} Z^{i} \stackrel{\partial^{i}}{\longrightarrow} Z^{i+1} \stackrel{\partial^{i+1}}{\longrightarrow} Z^{i+2} \longrightarrow \cdots
$$

where the images $I^{i}$ of the maps $\partial^{i}: Z^{i} \longrightarrow Z^{i+1}$ are all flat $R$-modules.

Remark 8.7. This category has been studied before; the reader can find the implication (ii) $\Longleftrightarrow$ (iii) in [5, Theorem 2.4, (1) $\Longleftrightarrow$ (3)]. The remainder of [5, Theorem 2.4] gives further characterizations of this category; what seems completely new is the description given in (i).

Proof. Lemma 8.5 showed that (i) $\Longrightarrow$ (ii). To prove that (ii) $\Longrightarrow$ (iii), assume that $Z$ is a filtered direct limit of contractible chain complexes of projective modules. The complex $Z$ is obviously acyclic. Furthermore, for each contractible complex $C$ of projective $R$-modules and each $i \in \mathbb{Z}$, the image of the map $\partial^{i}: C^{i} \longrightarrow C^{i+1}$ is a direct summand of $C^{i}$, and hence is a projective $R$-module. It follows that the image of $\partial^{i}: Z^{i} \longrightarrow Z^{i+1}$ is a filtered direct limit of projective modules, and must therefore be flat.

It remains to prove (iii) $\Longrightarrow$ (i). We must show that every acyclic complex of flat modules, where the images of $\partial^{i}: Z^{i} \longrightarrow Z^{i+1}$ are all flat, lies in $\mathbf{K}(R \text {-Proj })^{\perp}$.

Let $Z$ be an acyclic complex of flat modules, where the images of $\partial^{i}: Z^{i} \longrightarrow Z^{i+1}$ are flat. Consider the full subcategory $\mathcal{R} \subset \mathbf{K}(R$-Flat $)$ defined by

$$
\mathrm{Ob}(\mathcal{R})=\left\{X \in \mathbf{K}(R \text {-Proj }) \mid \forall n \in \mathbb{Z}, \operatorname{Hom}\left(\Sigma^{n} X, Z\right)=0\right\} .
$$

The category $\mathcal{R}$ is a localizing subcategory of $\mathbf{K}(R$-Proj $)$. We recall: this means that $\mathcal{R}$ is a triangulated subcategory, and is closed in $\mathbf{K}(R$-Proj) under coproducts. We wish to show that $\mathbf{K}(R$-Proj $) \subset \mathcal{R}$; by Corollary 5.10 it suffices to prove that $T \subset \mathcal{R}$.

We must therefore show that, for any object $X \in T$ and any morphism $f: X \longrightarrow Z$, the map $f$ is null homotopic. Now $X$ is a complex

$$
\cdots \longrightarrow X^{i-1} \stackrel{\partial^{i-1}}{\longrightarrow} X^{i} \stackrel{\partial^{i}}{\longrightarrow} X^{i+1} \stackrel{\partial^{i+1}}{\longrightarrow} X^{i+2} \longrightarrow \cdots \text {. }
$$

The map $f: X \longrightarrow Z$ is a chain map

$$
\begin{aligned}
& \cdots \longrightarrow X^{i-1} \stackrel{\partial_{X}^{i-1}}{\longrightarrow} X^{i} \stackrel{\partial_{X}^{i}}{\longrightarrow} X^{i+1} \stackrel{\stackrel{\partial_{X}^{i+1}}{\longrightarrow}}{\longrightarrow} X^{i+2} \longrightarrow \cdots \\
& f^{i-1} \downarrow \quad f^{i} \downarrow \quad f^{i+1} \downarrow \quad f^{i+2} \downarrow \\
& \cdots \longrightarrow Z^{i-1} \underset{\partial_{Z}^{i-1}}{\longrightarrow} Z^{i} \underset{\partial_{Z}^{i}}{\longrightarrow} Z^{i+1} \underset{\partial_{Z}^{i+1}}{\longrightarrow} Z^{i+2} \longrightarrow \cdots
\end{aligned}
$$


and we want to produce a null homotopy. That is, we want to produce maps

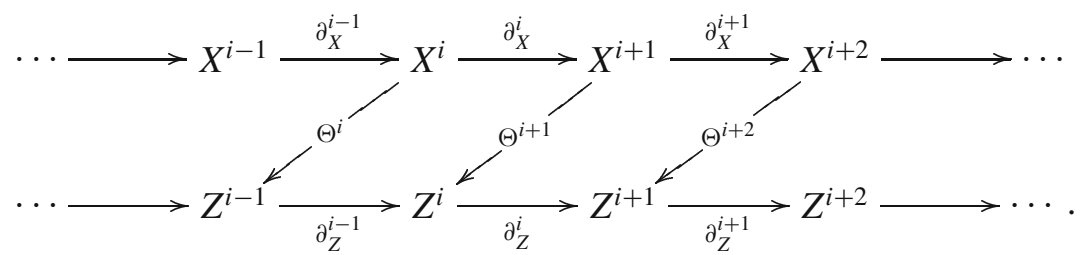

We will proceed to define the maps $\Theta^{i}: X^{i} \longrightarrow Z^{i-1}$ by induction on the integer $i$. Because $X \in T$, we know that $X^{i}=0$ if $i \ll 0$. We may choose an integer $j$ so that, if $i \leq j$, then $X^{i}=0$. For $i \leq j$ we define $\Theta^{i}=0$; we have no other choice.

Suppose $\Theta^{i}$ has been defined for $i \leq k$. Suppose further that, for $i<k$, we have

$$
f^{i}=\partial_{Z}^{i-1} \Theta^{i}+\Theta^{i+1} \partial_{X}^{i} .
$$

If $X^{i}=0$ for $i \leq k$, then this identity is automatic. We will show that we can extend the homotopy. Precisely, we can define $\Psi^{i}: X^{i} \longrightarrow Z^{i-1}$ for all $i \leq k+1$, so that

(i) $\Psi^{i}=\Theta^{i}$ for all $i \leq k-1$.

(ii) We have the identity

$$
f^{i}=\partial_{Z}^{i-1} \Psi^{i}+\Psi^{i+1} \partial_{X}^{i}
$$

for all $i<k+1$.

Note that we cannot guarantee that $\Theta^{k}=\Psi^{k}$; we might need to modify $\Theta^{k}$ to extend the homotopy. But for $i<k$ the $\Theta^{i}$ are stable.

It remains to prove the induction step. Suppose therefore that, for some integer $k$, we have defined $\Theta^{i}$ for all $i \leq k$, in such a way that

$$
f^{i}=\partial_{Z}^{i-1} \Theta^{i}+\Theta^{i+1} \partial_{X}^{i}
$$

for all $i<k$. Extend $\Theta$ to all $i$ by defining $\Theta^{i}=0$ for all $i>k$. Let $\bar{f}: X \longrightarrow Z$ be the chain map given by

$$
\bar{f}^{i}=f^{i}-\partial_{Z}^{i-1} \Theta^{i}-\Theta^{i+1} \partial_{X}^{i} .
$$

We have a chain map $\bar{f}: X \longrightarrow Z$. In the induction step we replace $f^{i}$ by $\bar{f}^{i}$, and $\Psi^{i}$ by $\bar{\Psi}^{i}=\Psi^{i}-\Theta^{i}$. The induction assertion becomes that, for all $i \leq k+1$, there exist maps $\dot{\Psi}^{i}: X^{i} \longrightarrow Z^{i-1}$, so that

(i') $\bar{\Psi}^{i}=0$ for all $i \leq k-1$.

(ii') We have the identity

$$
\bar{f}^{i}=\partial_{Z}^{i-1} \bar{\Psi}^{i}+\bar{\Psi}^{i+1} \partial_{X}^{i}
$$

for all $i<k+1$. 
Now observe that $\bar{f}: X \longrightarrow Z$ is a chain map from an object $X$ in $T$, that is from a complex of finitely generated projective $R$-modules, to a complex $Z$ of flat modules. Furthermore $\bar{f}^{i}=0$ for all $i<k$. Lemma 4.1 therefore applies; we can factor $\bar{f}$ as $X \stackrel{g}{\longrightarrow} Y \stackrel{h}{\longrightarrow} Z$, where $Y \in T$, and where $Y^{i}=0$ if $i<k$. The assertions ('') and (ii') above would immediately follow if we could produce, for every integer $i \leq k+1$, maps $\widetilde{\Psi}^{i}: Y^{i} \longrightarrow Z^{i-1}$, so that

(i”) $\widetilde{\Psi}^{i}=0$ for all $i \leq k-1$.

(ii') We have the identity

$$
h^{i}=\partial_{Z}^{i-1} \widetilde{\Psi}^{i}+\widetilde{\Psi}^{i+1} \partial_{Y}^{i}
$$

for all $i<k+1$.

Next note that $Y^{i}=0$ if $i<k$, which means that (i") has no choice but to be true. And furthermore, for $i<k$ the equality in (ii") is also immediate; there is only one map from $Y^{i}=0$ to $Z^{i}$. Therefore (i") and (ii") come down to showing that there exist two maps $\widetilde{\Psi}^{k}: Y^{k} \longrightarrow Z^{k-1}$ and $\widetilde{\Psi}^{k+1}: Y^{k+1} \longrightarrow Z^{k}$, satisfying the single identity

$$
h^{k}=\partial_{Z}^{k-1} \widetilde{\Psi}^{k}+\widetilde{\Psi}^{k+1} \partial_{Y}^{k}
$$

Next we prove the existence of the maps $\widetilde{\Psi}^{k}$ and $\widetilde{\Psi}^{k+1}$.

Consider therefore the chain map

$$
\begin{aligned}
& \cdots \quad 0 \longrightarrow Y^{k} \stackrel{\partial_{Y}^{k}}{\longrightarrow} Y^{k+1} \stackrel{\partial_{Y}^{k+1}}{\longrightarrow} Y^{k+2} \longrightarrow \cdots
\end{aligned}
$$

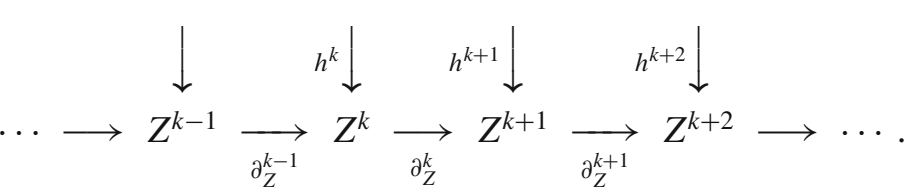

Let $I^{k+1}$ be the image of the map $\partial_{Z}^{k+1}: Z^{k+1} \longrightarrow Z^{k+2}$. The commutative diagram

$$
\begin{aligned}
& Y^{k} \stackrel{\partial_{Y}^{k}}{\longrightarrow} Y^{k+1} \\
& h^{k} \downarrow \quad h^{k+1} \downarrow \\
& Z^{k} \underset{\partial_{Z}^{k}}{\longrightarrow} Z^{k+1} \longrightarrow I^{k+1} \subset Z^{k+2}
\end{aligned}
$$

tells us that the composite

$$
\begin{aligned}
& Y^{k} \stackrel{\partial_{Y}^{k}}{\longrightarrow} Y^{k+1} \\
& h^{k+1} \downarrow \\
& Z^{k+1} \longrightarrow I^{k+1}
\end{aligned}
$$


must vanish. We have a vanishing composite $Y^{k} \longrightarrow Y^{k+1} \longrightarrow I^{k+1}$, with both $Y^{k}, Y^{k+1}$ finitely generated and projective, and with $I^{k+1}$ flat. Corollary 3.3 applies. It allows us to factor the map $Y^{k+1} \longrightarrow I^{k+1}$ as $Y^{k+1} \longrightarrow F \longrightarrow I^{k+1}$, with $F$ finitely generated and projective, and in such a way that the composite $Y^{k} \longrightarrow Y^{k+1} \longrightarrow F$ vanishes. In other words we can form a commutative diagram

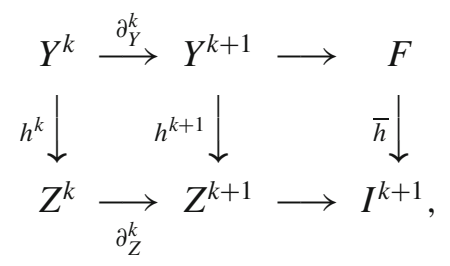

with $F$ finitely generated and projective, and where the horizontal composites vanish. We deduce a map of chain complexes

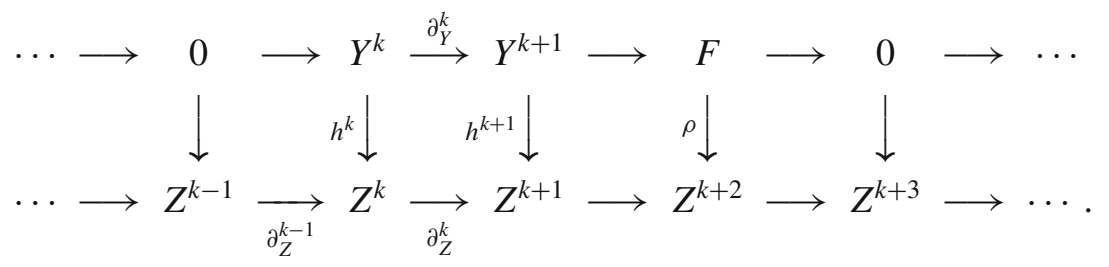

This is a map from a bounded above chain complex of projective modules to an acyclic complex $Z$, and must therefore be null homotopic. There is a homotopy

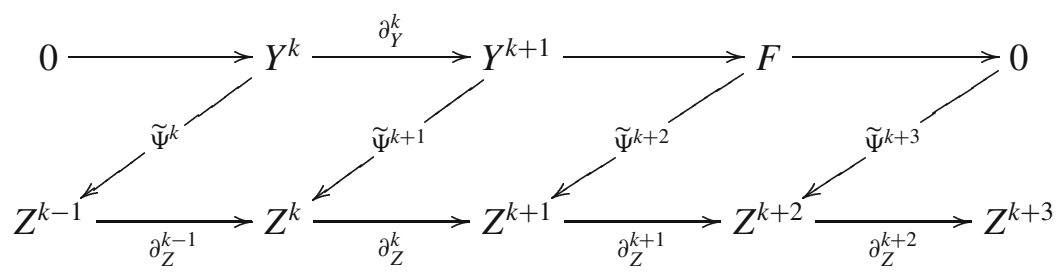

and the part that is relevant to our induction is that we have defined $\widetilde{\Psi}^{k}$ and $\widetilde{\Psi}^{k+1}$, so that

$$
h^{k}=\partial^{k-1} \widetilde{\Psi}^{k}+\widetilde{\Psi}^{k+1} \partial^{k} .
$$

This completes the induction.

Remark 8.8. Note that Theorem 8.6 gives us another way to prove that the functor $j^{*}: \mathbf{K}(R$-Flat $) \longrightarrow \mathbf{K}(R$-Proj $)$ preserves coproducts; we saw one proof in Remark 8.2. By Remark 2.12 we know that the functor $j^{*}$ can be identified with the Verdier quotient map $\pi: \mathbf{K}(R$-Flat $) \longrightarrow$ $\mathbf{K}(R$-Flat $) / \mathbf{K}(R \text {-Proj })^{\perp}$. The descriptions of the subcategory $\mathbf{K}(R \text {-Proj })^{\perp} \subset$ $\mathbf{K}(R$-Flat), given in Theorem 8.6(ii) or (iii), make it clear that it is closed under coproducts; from [11, Lemma 3.2.10] it follows that the functor $\pi$ respects coproducts. 


\section{The behavior of $\mathbf{K}(R \text {-Proj })^{\perp}$ under tensor product}

It is now time to understand how tensor product acts on the category $\mathbf{K}(R \text {-Proj })^{\perp}$. Given a dualizing complex $Y$, we are interested in the functor

$$
Y \otimes_{R}-: \mathbf{K}(R \text {-Flat }) \longrightarrow \mathbf{K}(R \text {-Inj }),
$$

that is the functor taking $Z$ to $Y \otimes_{R} Z$. To study it well, we have to understand what happens when $Z$ belongs to $\mathbf{K}(R \text {-Proj })^{\perp} \subset \mathbf{K}(R$-Flat $)$. We begin with general observations.

Proposition 9.1. Let $Z$ be an object in $\mathbf{K}(R \text {-Proj })^{\perp}$, and let $X$ be any complex of right $R$-modules. Then the tensor product $X \otimes_{R} Z$ is an acyclic complex.

Proof. By Theorem 8.6, we know that $Z$ is a filtered colimit of contractible complexes $Z_{\lambda}$. This makes $X \otimes_{R} Z$ the filtered colimit of the contractible complexes $X \otimes_{R} Z_{\lambda}$, hence acyclic.

Proposition 9.2. Let $Z$ be an object in $\mathbf{K}(R \text {-Proj })^{\perp}$, and let $X$ be any complex of $R-R$ bimodules. Then, for any finitely presented module $M$, the complex $\operatorname{Hom}_{R}\left(M, X \otimes_{R} Z\right)$ is acyclic.

Proof. Note that the module $M$ is finitely presented, while for each pair of integers $i, j \in \mathbb{Z}$, the module $Z^{j}$ is a flat $R$-module, and $X^{i}$ is an $R-R$ bimodule. Lemma 3.1 applies, and tells us that the natural map

$$
\varphi: \operatorname{Hom}_{R}\left(M, X^{i}\right) \otimes_{R} Z^{j} \longrightarrow \operatorname{Hom}_{R}\left(M, X^{i} \otimes_{R} Z^{j}\right)
$$

is an isomorphism. Also

$$
\begin{aligned}
\bigoplus_{i+j=n} \operatorname{Hom}_{R}\left(M, X^{i}\right) \otimes_{R} Z^{j} & \cong \bigoplus_{i+j=n} \operatorname{Hom}_{R}\left(M, X^{i} \otimes_{R} Z^{j}\right) \\
& \cong \operatorname{Hom}_{R}\left(M, \bigoplus_{i+j=n} X^{i} \otimes_{R} Z^{j}\right),
\end{aligned}
$$

where the last isomorphism is because $M$ is finitely presented. It follows that the natural map of complexes

$$
\mathcal{H o m}_{R}(M, X) \otimes_{R} Z \longrightarrow \mathcal{H o m}_{R}\left(M, X \otimes_{R} Z\right)
$$

is an isomorphism; we have just shown that it is an isomorphism in each degree.

Now Proposition 9.1 tells us that the complex $\operatorname{Hom}_{R}(M, X) \otimes_{R} Z$ is acyclic. Hence the isomorphic complex $\mathcal{H}_{R}\left(M, X \otimes_{R} Z\right)$ must also be acyclic.

A somewhat restricted version of the next result may be found in Jørgensen [8]; see, in particular, the proof of Theorem 2.4. 
Lemma 9.3. Let $Z$ be a complex of flat R-modules. Suppose that, for every finitely presented $R$-module $M$, the complex $\operatorname{Hom}_{R}(M, Z)$ is acyclic. Then $Z$ lies in $\mathbf{K}(R \text {-Proj })^{\perp}$.

Proof. First note that, if we take $M=R$, then the complex $\mathcal{H o m}_{R}(R, Z)$ is naturally isomorphic to $Z$. Hence $Z \simeq \mathscr{H}_{R}(R, Z)$ is acyclic.

Next we will prove that the images $I^{i}$, of the maps $\partial^{i}: Z^{i} \longrightarrow Z^{i+1}$, are all flat. Fix the integer $i$, and the module $I^{i}$. The module $I^{i}$ is the filtered colimit of the finitely presented modules mapping to it. Let $M \longrightarrow I^{i}$ be a map from a finitely presented $R$-module to $I^{i}$. Because $\mathcal{H}_{R}(M, Z)$ is acyclic, the map must factor as $M \longrightarrow Z^{i} \longrightarrow I^{i}$. But now $M$ is finitely presented and $Z^{i}$ is flat, so Lemma 3.2 applies. The map $M \longrightarrow Z^{i}$ must factor as $M \longrightarrow F \longrightarrow Z^{i}$, with $F$ finitely generated and projective. We conclude that any map $M \longrightarrow I^{i}$ factors through a finitely generated, projective module $F$. This means that the maps $F \longrightarrow I^{i}$, from finitely generated projective modules $F$ to the fixed $I^{i}$, are cofinal in the filtered category of all maps $M \longrightarrow I^{i}$, with $M$ finitely presented. Thus $I^{i}$ is a filtered colimit of projective modules, hence flat.

This makes $Z$ an acyclic chain complex of flat modules, where the images of all the differentials $\partial^{i}: Z^{i} \longrightarrow Z^{i+1}$ are flat modules. By Theorem 8.6, we conclude that $Z$ belongs to $\mathbf{K}(R \text {-Proj })^{\perp}$.

Corollary 9.4. Let $Z$ be a complex of flat $R$-modules; that is, $Z$ is an object in $\mathbf{K}(R$-Flat). The following are equivalent:

(i) $Z$ lies in $\mathbf{K}(R \text {-Proj })^{\perp} \subset \mathbf{K}(R$-Flat $)$.

(ii) If $X$ is any chain complex of right $R$-modules, then $X \otimes_{R} Z$ is acyclic.

(iii) For all right $R$-modules $M$, the complex $M \otimes_{R} Z$ is acyclic.

(iv) For all finitely presented left $R$-modules $N$, the complex $\mathscr{H}_{\mathrm{om}_{R}}(N, Z)$ is acyclic.

Proof. The implication (i) $\Longrightarrow$ (ii) follows from Proposition 9.1; if $Z \in$ $\mathbf{K}(R \text {-Proj })^{\perp}$, then $X \otimes_{R} Z$ is acyclic.

The implication (ii) $\Longrightarrow$ (iii) is trivial.

The implication (iii) $\Longrightarrow$ (iv) follows from Lemma 4.2. Let $N$ be any finitely presented left $R$-module. By Lemma 4.2, the natural map

$$
\operatorname{Hom}_{R}(N, R) \otimes_{R} Z \longrightarrow \mathcal{H o m}_{R}(N, Z)
$$

is an isomorphism of chain complexes. Part (iii) tells us that the complex $\operatorname{Hom}_{R}(N, R) \otimes_{R} Z$ is acyclic; hence so is $\mathscr{H}_{R}(N, Z)$.

Finally we have to prove (iv) $\Longrightarrow$ (i). Here we use Lemma 9.3; since $\mathcal{H}_{R}(N, Z)$ is acyclic for all finitely presented $N$, Lemma 9.3 tells us that $Z$ must lie in $\mathbf{K}(R \text {-Proj })^{\perp}$.

Corollary 9.5. Let $X$ be a complex of $R-R$ bimodules. Suppose that, as left $R$-modules, all the $X^{i}$ are flat. Then we have 
(i) If $Z$ is any object in $\mathbf{K}\left(R\right.$-Flat), then so is $X \otimes_{R} Z$.

(ii) If $Z$ lies in $\mathbf{K}(R \text {-Proj })^{\perp} \subset \mathbf{K}(R$-Flat $)$, then $X \otimes_{R} Z$ also lies in $\mathbf{K}(R \text {-Proj })^{\perp}$.

Proof. Part (i) is obvious.

We prove (ii). Suppose therefore that $Z$ lies in $\mathbf{K}(R \text {-Proj })^{\perp} \subset \mathbf{K}(R$-Flat $)$. By Proposition 9.2 we know that $\operatorname{Hom}_{R}\left(M, X \otimes_{R} Z\right)$ is acyclic for all finitely presented $M$. By (i) we know that $X \otimes_{R} Z$ is a chain complex of flat $R$-modules. Combining these two facts, Lemma 9.3 permits us to conclude that $X \otimes_{R} Z$ is an object of $\mathbf{K}(R \text {-Proj })^{\perp}$.

Remark 9.6. All of this becomes simpler if the ring $R$ is commutative. In that case all left $R$-modules may be viewed as $R-R$ bimodules. If $X$ and $Z$ are chain complexes of flat $R$-modules, then so is the tensor product $X \otimes_{R} Z$. That is, the tensor product of complexes defines a symmetric monoidal structure on the category $\mathbf{K}(R$-Flat $)$. The category $\mathbf{K}(R$-Flat $)$ is a tensor triangulated category, in the terminology of Balmer [1,2]. The statement of Corollary 9.5(ii), in the case where $R$ is commutative, simplifies to saying that the subcategory $\mathbf{K}(R \text {-Proj })^{\perp} \subset \mathbf{K}(R$-Flat $)$ is a tensor ideal. That is, if $X \in \mathbf{K}(R$-Flat $)$ and $Z \in \mathbf{K}(R \text {-Proj })^{\perp}$, then $X \otimes_{R} Z \in \mathbf{K}(R \text {-Proj })^{\perp}$.

So far our theorems have been true for arbitrary rings $R$. In the case of commutative rings Corollary 9.5 simplifies somewhat, as observed in Remark 9.6; but at the core our treatment has been general. The next result is different; it only holds for Noetherian rings. We give the argument in some detail, to highlight the points at which we rely on the fact that $R$ is Noetherian.

Corollary 9.7. Let $R$ be a Noetherian ring (meaning left Noetherian). Let $X$ be a chain complex of $R-R$ bimodules, and assume that each $X^{i}$ is injective as a left $R$-module. Then we have

(i) If $Z$ is any object of $\mathbf{K}$ ( $R$-Flat), then $X \otimes_{R} Z$ belongs to $\mathbf{K}(R$-Inj). That is, $X \otimes_{R} Z$ is a chain complex of injective left $R$-modules.

(ii) If $Z$ lies in the subcategory $\mathbf{K}(R \text {-Proj })^{\perp} \subset \mathbf{K}(R$-Flat $)$, then $X \otimes_{R} Z$ is contractible.

Proof. We prove (i) first; that is we let $Z$ be any object of $\mathbf{K}$ ( $R$-Flat). For each integer $j$, the module $Z^{j}$ is flat; it may be written as a filtered direct limit of finitely generated, projective modules $F_{\lambda}$. The bimodules $X^{i}$ are injective as left $R$-modules. For each pair of integers $i, j \in \mathbb{Z}$, the module $X^{i} \otimes_{R} Z^{j}$ is a filtered direct limit of $X^{i} \otimes_{R} F_{\lambda}$, each of which is injective. Since the ring $R$ is Noetherian, it follows that $X^{i} \otimes_{R} Z^{j}$ is injective. Using again the fact that $R$ is Noetherian, we deduce that

$$
\bigoplus_{i+j=n} X^{i} \otimes_{R} Z^{j}
$$


is injective. Therefore the complex $X \otimes_{R} Z$ is a chain complex of injective $R$-modules. This completes the proof of (i).

It remains to prove (ii); assume therefore that $Z$ lies in $\mathbf{K}(R \text {-Proj })^{\perp} \subset$ $\mathbf{K}(R$-Flat). Proposition 9.2 tells us that, for every finitely presented module $M$, the complex

$$
\mathscr{H o m}\left(M, X \otimes_{R} Z\right)
$$

is acyclic. By (i) we know that $X \otimes_{R} Z$ is a complex of injective $R$-modules. Combining these two facts, and using yet again the assumption that $R$ is Noetherian, [9, Lemma 2.2] permits us to conclude that the complex $X \otimes_{R} Z$ must be contractible.

This concludes the proofs of all the facts which the introduction promised to deliver in this article.

\section{Appendix A. When right adjoints respect coproducts}

In this section we give a large-cardinal generalization of [10, Theorem 5.1]. It is a result which allows one to verify, quite easily in some cases, that the right adjoint of some triangulated functor respects coproducts. See Remark 8.2

Proposition A.1. Let \& be a [TR5] triangulated category, that is \& has arbitrary small coproducts. Let $T$ be an $\alpha$-compact generating set for $\&$, where $\alpha$ is some regular cardinal. Let $j_{!}: \& \longrightarrow \mathcal{T}$ be a triangulated functor, and suppose that $j_{!}$takes coproducts in $\&$ to coproducts in $\mathcal{T}$. The first assertion of the lemma is that $j_{1}: \& \longrightarrow \mathcal{T}$ has a right adjoint $j^{*}: \mathcal{T} \longrightarrow 8$.

Assume further that:

(i) The functor $j_{1}: \& \longrightarrow \mathcal{T}$ takes $T \subset \&$ to an $\alpha$-perfect set of $\alpha$-small objects in $\mathcal{T}$.

(ii) If $\left\{s_{\lambda}, \lambda \in \Lambda\right\}$ is any set of $<\alpha$ objects in $T$, then the natural map

$$
\coprod_{\lambda \in \Lambda} j^{*} j_{!} s_{\lambda} \longrightarrow j^{*}\left\{\coprod_{\lambda \in \Lambda} j_{!} s_{\lambda}\right\}
$$

is an isomorphism.

Then the functor $j^{*}: \mathcal{T} \longrightarrow \&$ respects coproducts.

Proof. First note that the functor $j_{1}: \& \longrightarrow \mathcal{T}$ is triangulated and respects coproducts. Next observe that $T$ is an $\alpha$-compact generating set for $\&$. From [11, Theorem 8.3.3] it follows that $\delta$ satisfies Brown representability, and [11, Theorem 8.4.4] permits us to conclude that $j_{!}: \& \longrightarrow \mathcal{T}$ has a right adjoint, which we will call $j^{*}: \mathcal{T} \longrightarrow \&$. 
It remains to show that this adjoint respects coproducts. For every set $\left\{X_{\lambda}, \lambda \in \Lambda\right\}$ of objects in $\mathcal{T}$, we need to show that the natural map

$$
\coprod_{\lambda \in \Lambda} j^{*} X_{\lambda} \longrightarrow j^{*}\left\{\coprod_{\lambda \in \Lambda} X_{\lambda}\right\}
$$

is an isomorphism. If we complete this map to a triangle

$$
\coprod_{\lambda \in \Lambda} j^{*} X_{\lambda} \longrightarrow j^{*}\left\{\coprod_{\lambda \in \Lambda} X_{\lambda}\right\} \longrightarrow Z \longrightarrow \Sigma \coprod_{\lambda \in \Lambda} j^{*} X_{\lambda}
$$

we need to show that $Z=0$. Equivalently, we need to show that, for every object $s \in T$, we have $\operatorname{Hom}(s, Z)=0$. From the long exact sequence of $\operatorname{Hom}(s,-)$ this comes down to showing that, for every integer $n \in \mathbb{Z}$ and every object $s \in T$, the natural map

(*) $\operatorname{Hom}_{s}\left(s, \coprod_{\lambda \in \Lambda} j^{*} \Sigma^{n} X_{\lambda}\right) \longrightarrow \operatorname{Hom}_{s}\left(s, j^{*}\left\{\coprod_{\lambda \in \Lambda} \Sigma^{n} X_{\lambda}\right\}\right)$

is an isomorphism. Without loss of generality we may assume $n=0$, and we may fix the set $\left\{X_{\lambda}, \lambda \in \Lambda\right\}$ of objects of $\mathcal{T}$. We will now prove that the map in $(*)$ is injective and surjective. We prove the surjectivity first.

Suppose we are given a map

$$
s \stackrel{\varphi}{\longrightarrow} j^{*}\left\{\coprod_{\lambda \in \Lambda} X_{\lambda}\right\} .
$$

By adjunction it gives a map

$$
j_{!} s \longrightarrow \coprod_{\lambda \in \Lambda} X_{\lambda}
$$

and because $j_{!} T$ is an $\alpha$-perfect set of $\alpha$-small objects in $\mathcal{T}$, this map factors as

$$
j_{!} s \longrightarrow \coprod_{\lambda \in \Lambda^{\prime}} j_{!} s_{\lambda} \stackrel{\amalg_{\lambda \in \Lambda^{\prime}} f_{\lambda}}{\longrightarrow} \coprod_{\lambda \in \Lambda^{\prime}} X_{\lambda} \subset \coprod_{\lambda \in \Lambda} X_{\lambda} .
$$

In this factorization the set $\Lambda^{\prime} \subset \Lambda$ has cardinality $<\alpha$, and the objects $s_{\lambda}$ lie in $T$. The map $\varphi$ can be recovered as the composite

$$
s \stackrel{\eta_{s}}{\longrightarrow} j^{*} j_{!} s \longrightarrow j^{*} \coprod_{\lambda \in \Lambda^{\prime}} j_{!} s_{\lambda} \stackrel{j^{*} \amalg_{\lambda \in \Lambda^{\prime}} f_{\lambda}}{\longrightarrow} j^{*}\left\{\coprod_{\lambda \in \Lambda} X_{\lambda}\right\}
$$

where $\eta_{s}: s \longrightarrow j^{*} j_{!} s$ is the unit of adjunction. Hypothesis (ii) of the proposition says that $j^{*} \bigsqcup_{\lambda \in \Lambda^{\prime}} j_{!} s_{\lambda}=\bigsqcup_{\lambda \in \Lambda^{\prime}} j^{*} j_{!} s_{\lambda}$. The factorization can therefore be rewritten as

$$
s \stackrel{\eta_{s}}{\longrightarrow} j^{*} j_{!} s \longrightarrow \coprod_{\lambda \in \Lambda^{\prime}} j^{*} j_{!} s_{\lambda} \longrightarrow j^{*}\left\{\coprod_{\lambda \in \Lambda} X_{\lambda}\right\}
$$


and this obviously factors through

$$
s \stackrel{\eta_{s}}{\longrightarrow} j^{*} j_{!} s \longrightarrow \coprod_{\lambda \in \Lambda^{\prime}} j^{*} j_{!} s_{\lambda} \stackrel{\amalg_{\lambda \in \Lambda^{\prime}} j^{*} f_{\lambda}}{\longrightarrow} \coprod_{\lambda \in \Lambda} j^{*} X_{\lambda} \longrightarrow j^{*}\left\{\coprod_{\lambda \in \Lambda} X_{\lambda}\right\}
$$

proving the surjectivity.

Next we show the injectivity. Suppose therefore that we have a map $\theta: s \longrightarrow \bigsqcup_{\lambda \in \Lambda} j^{*} X_{\lambda}$, so that the composite

$$
s \stackrel{\theta}{\longrightarrow} \coprod_{\lambda \in \Lambda} j^{*} X_{\lambda} \longrightarrow j^{*}\left\{\coprod_{\lambda \in \Lambda} X_{\lambda}\right\}
$$

vanishes; we need to show that $\theta$ vanishes. In any case $\theta$ must factor as

$$
s \longrightarrow \coprod_{\lambda \in \Lambda^{\prime}} s_{\lambda} \stackrel{\amalg_{\lambda \in \Lambda^{\prime}} f_{\lambda}}{\longrightarrow} \coprod_{\lambda \in \Lambda} j^{*} X_{\lambda}
$$

with $\Lambda^{\prime} \subset \Lambda$ of cardinality $<\alpha$, and with $s_{\lambda} \in T$. The vanishing of

$$
s \longrightarrow \coprod_{\lambda \in \Lambda^{\prime}} s_{\lambda} \stackrel{\amalg_{\lambda \in \Lambda^{\prime}} f_{\lambda}}{\longrightarrow} \coprod_{\lambda \in \Lambda} j^{*} X_{\lambda} \longrightarrow j^{*}\left\{\coprod_{\lambda \in \Lambda} X_{\lambda}\right\}
$$

implies, by adjunction, the vanishing of the composite

$$
j_{!} s \longrightarrow j_{!} \coprod_{\lambda \in \Lambda^{\prime}} s_{\lambda} \stackrel{j_{!} \amalg_{\lambda \in \Lambda^{\prime}} f_{\lambda}}{\longrightarrow} j_{!} \coprod_{\lambda \in \Lambda} j^{*} X_{\lambda} \longrightarrow \coprod_{\lambda \in \Lambda} X_{\lambda}
$$

but $j_{\text {! }}$ commutes with all coproducts, by hypothesis. We can write this vanishing composite as

$$
j_{!} s \longrightarrow \coprod_{\lambda \in \Lambda^{\prime}} j_{!} s_{\lambda} \stackrel{\bigsqcup_{\lambda \in \Lambda^{\prime}} j_{!} f_{\lambda}}{\longrightarrow} \coprod_{\lambda \in \Lambda} j_{!} j^{*} X_{\lambda} \longrightarrow \coprod_{\lambda \in \Lambda} X_{\lambda}
$$

Now recall that $j_{!} T$ is an $\alpha$-perfect set of $\alpha$-small objects in $\mathcal{T}$. Hence each map

$$
j_{!} s_{\lambda} \stackrel{j_{!} f_{\lambda}}{\longrightarrow} j_{!} j^{*} X_{\lambda} \stackrel{\varepsilon_{X}}{\longrightarrow} X_{\lambda}
$$

can be factored as

$$
j_{!} s_{\lambda} \stackrel{g_{\lambda}}{\longrightarrow} j_{!} t_{\lambda} \stackrel{h_{\lambda}}{\longrightarrow} X_{\lambda}
$$

so that $t_{\lambda} \in T$, and so that the composite

$$
j_{!} s \longrightarrow \coprod_{\lambda \in \Lambda^{\prime}} j_{!} s_{\lambda} \stackrel{\amalg_{\lambda \in \Lambda^{\prime}} g_{\lambda}}{\longrightarrow} \coprod_{\lambda \in \Lambda^{\prime}} j_{!} t_{\lambda}
$$


vanishes. This means that the composite

$$
s \stackrel{\eta_{s}}{\longrightarrow} j^{*} j_{!} s \longrightarrow j^{*} \coprod_{\lambda \in \Lambda^{\prime}} j_{!} s_{\lambda} \stackrel{j^{*} \amalg_{\lambda \in \Lambda^{\prime}} g_{\lambda}}{\longrightarrow} j^{*} \coprod_{\lambda \in \Lambda^{\prime}} j_{!} t_{\lambda}
$$

must also vanish. But Hypothesis (ii) of the Proposition guarantees that $j^{*} \coprod_{\lambda \in \Lambda^{\prime}} j_{!} s_{\lambda}=\bigsqcup_{\lambda \in \Lambda^{\prime}} j^{*} j_{!} s_{\lambda}$, and that $j^{*} \coprod_{\lambda \in \Lambda^{\prime}} j_{!} t_{\lambda}=\coprod_{\lambda \in \Lambda^{\prime}} j^{*} j_{!} t_{\lambda}$. We deduce that the composite

$$
s \stackrel{\eta_{s}}{\longrightarrow} j^{*} j_{!} s \longrightarrow \coprod_{\lambda \in \Lambda^{\prime}} j^{*} j_{!} s_{\lambda} \stackrel{\amalg_{\lambda \in \Lambda^{\prime}} j^{*} g_{\lambda}}{\longrightarrow} \coprod_{\lambda \in \Lambda^{\prime}} j^{*} j_{!} t_{\lambda}
$$

vanishes, and hence so does the longer composite

$$
s \stackrel{\eta_{s}}{\longrightarrow} j^{*} j_{!} s \longrightarrow \coprod_{\lambda \in \Lambda^{\prime}} j^{*} j_{!} s_{\lambda} \stackrel{\amalg_{\lambda \in \Lambda^{\prime}} j^{*} g_{\lambda}}{\longrightarrow} \coprod_{\lambda \in \Lambda^{\prime}} j^{*} j_{!} t_{\lambda} \stackrel{\amalg_{\lambda \in \Lambda^{\prime}} j^{*} h_{\lambda}}{\longrightarrow} \coprod_{\lambda \in \Lambda} j^{*} X_{\lambda},
$$

which is nothing other than the map $\theta$.

\section{References}

1. Balmer, P.: Presheaves of triangulated categories and reconstruction of schemes. Math. Ann. 324(3), 557-580 (2002)

2. Balmer, P.: The spectrum of prime ideals in tensor triangulated categories. J. Reine Angew. Math. 588, 149-168 (2005)

3. Bökstedt, M., Neeman, A.: Homotopy limits in triangulated categories. Compos. Math. 86, 209-234 (1993)

4. Bousfield, A.K.: The localization of spectra with respect to homology. Topology 18, 257-281 (1979)

5. Enochs, E.E., García Rozas, J.R.: Flat covers of complexes. J. Algebra 210(1), 86-102 (1998)

6. Gabriel, P., Zisman, M.: Calculus of Fractions and Homotopy Theory. Ergeb. Math. Grenzgeb., vol. 35. Springer, New York (1967)

7. Iyengar, S., Krause, H.: Acyclicity versus total acyclicity for complexes over Noetherian rings. Doc. Math. 11, 207-240 (2006)

8. Jørgensen, P.: The homotopy category of complexes of projective modules. Adv. Math. 193(1), 223-232 (2005)

9. Krause, H.: The stable derived category of a Noetherian scheme. Compos. Math. 141(5), 1128-1162 (2005)

10. Neeman, A.: The Grothendieck duality theorem via Bousfield's techniques and Brown representability. J. Am. Math. Soc. 9, 205-236 (1996)

11. Neeman, A.: Triangulated Categories. Ann. Math. Stud., vol. 148. Princeton University Press, Princeton, NJ (2001)

12. Verdier, J.-L.: Des catégories dérivées des catégories abeliennes. Astérisque, vol. 239. Société Mathématique de France, Paris (1996) 